\title{
Correlated Disruption of Resting-State fMRI, LFP, and Spike Connectivity between Area 3b and S2 following Spinal Cord Injury in Monkeys
}

\author{
-Ruiqi Wu, ${ }^{1,2,4}$ ๑Pai-Feng Yang, ${ }^{1,2}$ and Li Min Chen ${ }^{1,2,3}$ \\ ${ }^{1}$ Department of Radiology and Radiological Sciences, ${ }^{2}$ Institute of Imaging Science, and ${ }^{3}$ Department of Psychology, Vanderbilt University, Nashville, \\ Tennessee, 37232, and ${ }^{4}$ Wuhan Institute of Physics and Mathematics, Chinese Academy of Sciences/State Key Laboratory of Magnetic Resonance and \\ Atomic and Molecular Physics, Wuhan, Hubei, 430071, China
}

This study aims to understand how functional connectivity (FC) between areas 3b and S2 alters following input deprivation and the neuronal basis of disrupted FC of resting-state fMRI signals. We combined submillimeter fMRI with microelectrode recordings to localize the deafferented digit regions in areas $3 \mathrm{~b}$ and $\mathrm{S} 2$ by mapping tactile stimulus-evoked fMRI activations before and after cervical dorsal column lesion in each male monkey. An average afferent disruption of $97 \%$ significantly reduced fMRI, local field potential (LFP), and spike responses to stimuli in both areas. Analysis of resting-state fMRI signal correlation, LFP coherence, and spike cross-correlation revealed significantly reduced functional connectivity between deafferented areas $3 \mathrm{~b}$ and S2. The degrees of reductions in stimulus responsiveness and FC after deafferentation differed across fMRI, LFP, and spiking signals. The reduction of FC was much weaker than that of stimulus-evoked responses. Whereas the largest stimulus-evoked signal drop $(\sim 80 \%)$ was observed in LFP signals, the greatest FC reduction was detected in the spiking activity $(\sim 30 \%)$. $\mathrm{fMRI}$ signals showed mild reductions in stimulus responsiveness $(\sim 25 \%)$ and $\mathrm{FC}$ $(\sim 20 \%)$. The overall deafferentation-induced changes were quite similar in areas $3 \mathrm{~b}$ and S2 across signals. Here we demonstrated that FC strength between areas $3 \mathrm{~b}$ and S2 was much weakened by dorsal column lesion, and stimulus response reduction and FC disruption in fMRI covary with those of LFP and spiking signals in deafferented areas $3 \mathrm{~b}$ and S2. These findings have important implications for fMRI studies aiming to probe FC alterations in pathological conditions involving deafferentation in humans.

Key words: electrophysiology; functional connectivity; fMRI; nonhuman primate; spinal cord injury

\section{Significance Statement}

By directly comparing fMRI, local field potential, and spike signals in both tactile stimulation and resting states before and after severe disruption of dorsal column afferent, we demonstrated that reduction in fMRI responses to stimuli is accompanied by weakened resting-state fMRI functional connectivity (FC) in input-deprived and reorganized digit regions in area $3 \mathrm{~b}$ of the $\mathrm{S} 1$ and S2. Concurrent reductions in local field potential and spike FC validated the use of resting-state fMRI signals for probing neural intrinsic FC alterations in pathological deafferented cortex, and indicated that disrupted FC between mesoscale functionally highly related regions may contribute to the behavioral impairments.

\section{Introduction}

Since the discovery of highly correlated fluctuations of restingstate fMRI ( rsfMRI) signals between distant brain regions, it has been widely used as an indicator of intrinsic interareal functional

\footnotetext{
Received Aug. 15, 2017; revised 0ct. 2, 2017; accepted 0ct. 4, 2017

Author contributions: L.M.C. designed research; R.W. and P.-F.Y. performed research; R.W. analyzed data; R.W. and L.M.C. wrote the paper.

This work was supported by the Dana Foundation to L.M.C., and National Institutes of Health Grant R01 NS069909 to L.M.C. We thank Dr. Robert M. Friedman, Tung-Lin Wu, and Zhaoyue Shi for assistance on electrophysiological data collection; Feng Wang and Fuxue Xin for assistance on fMRI data collection; Chaohui Tang for technical support on animal preparation; and George Wilson, III, for language editing of the manuscript.

The authors declare no competing financial interests.
}

connectivity (FC) (Biswal et al., 1995; Raichle, 2010). fMRI resting-state $\mathrm{FC}$ ( $\mathrm{rsFC}$ ) alterations have been proposed to be noninvasive and sensitive biomarkers for many pathological conditions, including schizophrenia, autism, stroke, and chronic pain (Rotarska-Jagiela et al., 2010; Wang et al., 2017; Yang et al., 2017). rsFC has been widely used for probing functional circuit changes in clinical populations (Fox and Greicius, 2010). Despite its wide

Correspondence should be addressed to Dr. Li Min Chen, Department of Radiology and Radiological Sciences, Institute of Imaging Science, Vanderbilt University Medical Center, 1105 MCN, 116121 st Avenue South, Nashville, TN 37232. E-mail: limin.chen@vanderbilt.edu.

DOI:10.1523/JNEUROSCI.2318-17.2017

Copyright $\odot 2017$ the authors $\quad 0270-6474 / 17 / 3711192-12 \$ 15.00 / 0$ 
application, the neural underpinnings of the rsFC signals, particularly in pathological conditions, have not been well established. It prohibits a full appreciation of the clinical significance of the rsFC changes observed in many disease conditions. Direct comparisons of rsfMRI and electrophysiological signals before and after targeted disruption of ascending dorsal column afferents provide critical information for improving our understating of the rsFC alterations in similar pathological conditions in humans. Highly synchronized oscillations of the spontaneous local field potential (LFP) or spiking activities between brain regions indicate strong functional connection (Eckhorn et al., 1988; Plewnia et al., 2008; Reed et al., 2008; Wilson et al., 2016). Our recent discoveries of dynamic functional organization in somatic areas $3 \mathrm{~b}, 1$, and secondary somatosensory cortex (S2) and significantly altered weakened horn-to-horn rsFC in injured spinal cord gray matter, and its recovery over time underscored the behavioral significance of changes in region-to-region rsFC (Yang et al., 2014; Chen et al., 2015). Previous studies have demonstrated strong correlative relationships between electrophysiological and BOLD signals under processing of sensory inputs (Logothetis et al., 2001; Mukamel et al., 2005; Ekstrom, 2010; Wang et al., 2013). Here we compared directly their relationships during stimulating and resting states.

Specifically, we aim to address several questions: Does dorsal column input deprivation to cortex alter the FC between areas $3 \mathrm{~b}$ and S2? Do FC changes measured by rsfMRI agree with those measured by spontaneous LFP and spiking activity, and if so, in what manner? We chose the digit representations in areas $3 \mathrm{~b}$ and S2 in monkeys and the nearly complete unilateral dorsal column lesion (DCL) as our experimental model for several reasons. First, both areas $3 \mathrm{~b}$ and S2 receive ascending somatosensory inputs from the thalamus. Much of the outputs of area $3 \mathrm{~b}$ project to high-order S2 cortex (Krubitzer and Kaas, 1992; Kaas, 1993). Their well-characterized anatomical connections make them well suited to test the hypothesis that shared common inputs (e.g., from subcortical regions) are one of the driving forces of the synchronized fluctuation of rsfMRI signals (van den Heuvel and Hulshoff Pol, 2010). Second, proper functional interactions between area $3 \mathrm{~b}$ and S2 neurons are critical for the execution of touch perception and hand use. Anatomical and electrophysiological evidence indicates that areas 3b, 1, and S2 undergo reactivation and reorganization processes after DCL, and these plastic changes mediate functional and behavioral recovery (Chen et al., 2012; Yang et al., 2014). Third, decreased fMRI rsFC between S1 and S2 was found in patients with spinal cord injury (SCI), but increased brain rsFC between sensorimotor and visual networks was revealed (Choe et al., 2013; Min et al., 2015). An fMRI study in monkeys showed that fractional amplitude of low-frequency fluctuations varies significantly in several encephalic regions following SCI (Rao et al., 2014). Last, our previous studies have demonstrated that submillimeter resolution fMRI is capable of detecting DCL-induced fMRI activation changes in signal location and magnitude in areas 3b, 1, and S2 (Chen et al., 2012; Yang et al., 2014).

This study aims to (1) characterize the properties of hemodynamic and neuronal connectivity between normal areas $3 \mathrm{~b}$ and S2; (2) examine the effects of input deprivation caused by DCL on the hemodynamic and neuronal connectivity between areas $3 \mathrm{~b}$ and S2; and (3) determine the relationship between alterations of stimulus-evoked responses and rsFC by combining fMRI and imaged-guided microelectrode recordings in monkeys during normal versus SCI conditions.

\section{Materials and Methods}

Animal preparation and surgery. Six adult male squirrel monkeys (Saimiri sciureus) (SM-BUL, SM-GUA, SM-SUP, SM-CHA, SM-KEE, and SM4043) were included in this study. A unilateral DCL at the high cervical spinal cord level between vertebrae $\mathrm{C} 4$ and $\mathrm{C} 5$ was performed on 3 of the monkeys (SM-BUL, SM-GUA, and SM-SUP). fMRI data in both stimulation and resting states were collected on these 3 monkeys before and after DCL. In particular, to avoid the image distortion due to craniotomy, fMRI data were collected before animals underwent craniotomy in this study. Intracortical microelectrode electrophysiological recordings of LFP and spiking signals were performed on 3 DCL and 3 normal monkeys (SM-CHA, SM-KEE, and SM-4043).

For data collection, animals were initially sedated with ketamine hydrochloride $(10 \mathrm{mg} / \mathrm{kg})$ and atropine sulfate $(0.05 \mathrm{mg} / \mathrm{kg}$, i.m. $)$, and then were maintained with isoflurane $(0.5 \%-1.2 \%)$ delivered in a $70: 30$ $\mathrm{N}_{2} \mathrm{O} / \mathrm{O}_{2}$ mixture throughout the experiment. Animals were artificially ventilated to maintain an end-tidal $\mathrm{CO}_{2}$ of $\sim 4 \%$. Rectal temperature was maintained between $37.5^{\circ} \mathrm{C}$ and $38.5^{\circ} \mathrm{C}$ by means of a circulating water blanket. Heart rate, respiration pattern, saturation of peripheral oxygen, and ECG were also monitored and maintained at stable condition during the entire procedure. Detailed procedures have been described previously (Qi et al., 2011; Shi et al., 2017; Wu et al., 2017). All procedures were conducted in accordance with National Institutes of Health guidelines and approved by Animal Care and Use Committees at Vanderbilt University.

Stimulus presentation protocol. Hand and fingers were positioned to make the distal pads available for tactile stimulation. Pegs were glued to fingernails and then imbedded in plasticine to ensure a stable probe contact. Trapezoid tactile indentation (in $0.34 \mathrm{~mm}$ vertical displacement) was presented repeatedly on individual distal finger pads. Indentation was generated by a round plastic probe ( $2 \mathrm{~mm}$ in diameter) attached to a piezoelectric device (Noliac), which was driven by Grass S48 square wave stimulators (Grass-Telefactor). Within each fMRI experimental session (day), multiple runs of fMRI datasets lasting $\sim 10 \mathrm{~min}$ were obtained with and without (resting-state) tactile stimulation. To identify digit representations within S1 cortex, $30 \mathrm{~s}$ on/off cycles of $8 \mathrm{~Hz}$ tactile indentation were presented seven times within a single imaging run to elicit fMRI responses. For electrophysiological LFP and spike recording experiments, tactile indentation was presented repeatedly at a rate of $0.5 \mathrm{~s}$ of probe contact followed by a $2.0 \mathrm{~s}$ delay between stimulations for 100 trials per run. The timestamps of the stimulus on and off were recorded along with the spiking and LFP signals. Each stimulus presentation cycle was divided into two periods for quantification: $1 \mathrm{~s}$ prestimulus onset as resting period and $0.5 \mathrm{~s}$ during the stimulus as stimulating period. The probe was in light contact with the skin during the no stimulation periods.

MRI data acquisition and analysis. Each animal underwent multiple MRI scan sessions under anesthesia. We typically acquire structural MRI images first, then followed by fMRI scans. During structural MRI scans, animals were maintained at relatively high isoflurane level $(0.9 \%-1.2 \%)$. We lower the isoflurane level before fMRI scans, and maintained at a light and stable level $(\sim 0.7 \%-0.8 \%)$ during the functional data acquisition periods. The depth of anesthesia was carefully maintained by keeping the heart rate, end-tidal $\mathrm{CO}_{2}$, and chest movement pattern (via a piezoelectric stretch sensor placed under chest) at stable readings via fine-tuning the isoflurane level. All MRI scans were performed on a 9.4 T Inova magnet (Varian Medical Systems), using a 3-cm-diameter surface transmit-receive coil positioned over $\mathrm{S} 1$ (areas $3 \mathrm{a}, 3 \mathrm{~b}$, and $1 / 2$ ) and $\mathrm{S} 2$ cortices contralateral to the stimulated hand (Fig. $1 A, B$ ). Four 2-mm-thick oblique image slices were centered over the central and lateral sulci with the first slice covering S1 and the third slice covering S2 for MRI data acquisition (Fig. $1 B)$. High-resolution $\mathrm{T} 2^{\star}$-weighted gradient echo anatomical images $\left(\mathrm{TR}=200 \mathrm{~ms}, \mathrm{TE}=16 \mathrm{~ms}\right.$, and resolution $\left.=0.078 \times 0.078 \times 2 \mathrm{~mm}^{3}\right)$ were obtained. $\mathrm{PMRI}$ data were acquired using a gradient EPI sequence $\left(\mathrm{TR}=1500 \mathrm{~ms}, \mathrm{TE}=19 \mathrm{~ms}\right.$, and resolution $\left.=0.55 \times 0.55 \times 2 \mathrm{~mm}^{3}\right)$. Each fMRI run (with and without stimulation) consisted of 300 image volumes. After baseline (prelesion) fMRI data collection, 3 monkeys (SM-BUL, SM-GUA, and SM-SUP) received unilateral DCL. fMRI data collection was repeated after lesion. 
A

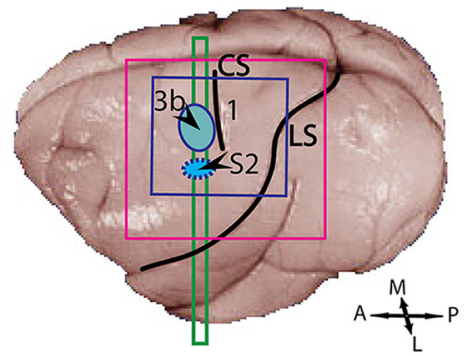

D

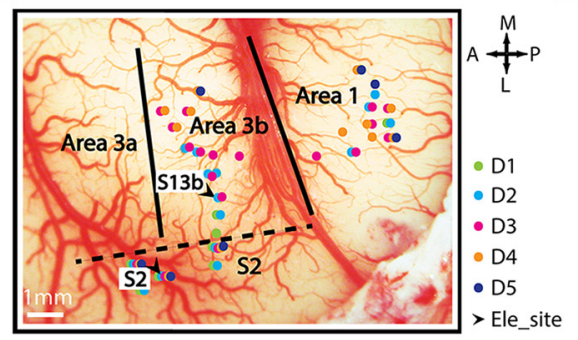

B

E
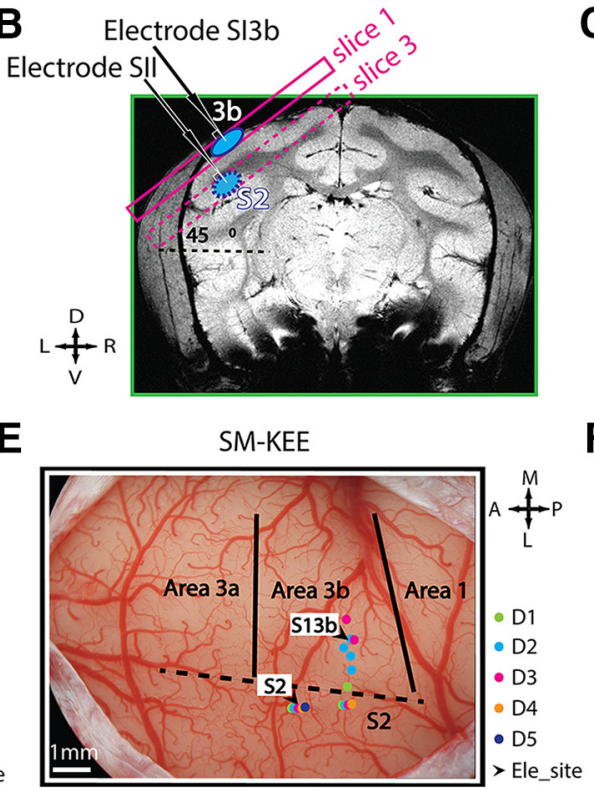

C

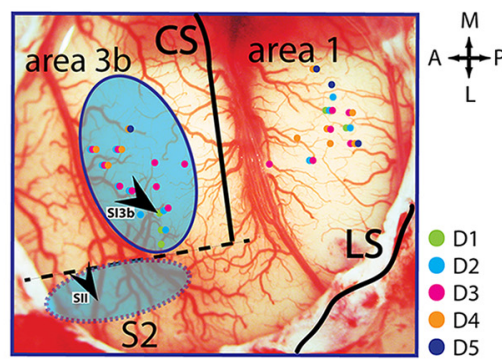

$\mathbf{F}$

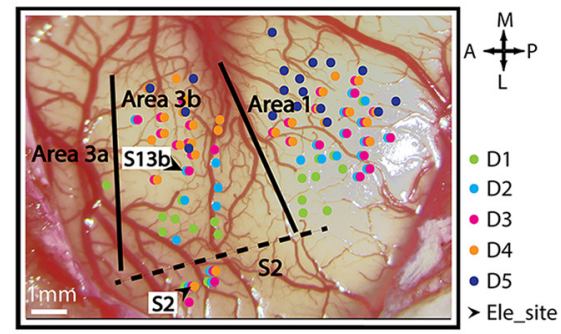

Figure 1. Experimental setup and illustration of microelectrode mapping and recording sites in areas 3b and S2 in 3 normal animals (SM-CHA, SM-KEE, and SM-4043). $A$, The locations (blue patches) of area $3 \mathrm{~b}$ and projected $S 2$ on the brain surface. Purple and blue squares represent the FOV of MRI image and blood vessel image for electrophysiology, respectively. CS, Central sulci; $L S$, lateral sulci; $A$, anterior; L, lateral; $M$, medial; P, posterior. $B$, Coronal T2-weighted structural image shows the placements of oblique MRI slices. Solid and dotted purple rectangle outlines represent slices 1 and 3 covering area $3 b$ and $S 2$ regions, respectively. Blue patches represent the locations of tactile activation. D, Dorsal; $V$, ventral; L, left; $R$, right. C, Methods for identifying area $3 b$ and $S 2$ recording sites. Color-coded dots on the corresponding blood vessel map indicate the microelectrode penetration sites and receptive field properties. Black arrows indicate recording sites in areas $3 \mathrm{~b}$ and S2. Green dots indicate D1 responsive sites. Cyan dots indicate D2 response sites. Purple dots indicate D3 sites. Orange dots indicate D4 response sites. Navy dots indicate D5 response sites. Black arrows indicate the chosen recording sites within each $\mathrm{fMRl}$ activation focus (blue patches). Recording sites were logged and illustrated on the corresponding blood vessel map in each animal. For SM-KEE (D), SM-CHA (E), and SM-4043 (F), tactile stimulus was presented on D2 or D3. Black arrows indicate the recording sites in area $3 \mathrm{~b}(700-900 \mu \mathrm{m}$ in depth) and area S2 (3500-5000 $\mu \mathrm{m}$ in depth). Electrode penetration sites (small colored dots) on each vessel map show the receptive field of different digits. Color-coded individual digit site is shown by the inset column in each animal. Green dots indicate D1. Cyan dots indicate D2. Purple dots indicate D3. Orange dots indicate D4. Navy dots indicate D5. A, Anterior; L, lateral; M, medial; P, posterior. Solid black lines indicate estimated interarea borders. Dotted black lines indicate estimated digit-face borders.

Functional EPI data were preprocessed with slice timing and head motion correction (3dTshift, AFNI, RRID:SCR_005927), physiological noise correction using RETROICOR algorithm (3dretroicor, AFNI), and spatial smoothing (Gaussian kernel FWHM $=1 \mathrm{~mm}$, 3dmerge, AFNI). The stimulus-evoked EPI data were temporally smoothed with a lowpass filter with a $0.25 \mathrm{~Hz}$ cutoff frequency, and the resting-state data with a $0.1 \mathrm{~Hz}$ cutoff frequency ( $3 \mathrm{dFourier}, \mathrm{AFNI}$ ). Activation maps were created by voxelwise correlation analysis, using the HRF convolved stimulus presentation paradigm (3dDeconvolve, AFNI) as a predictor, and were coregistered to the corresponding $\mathrm{T} 2^{\star}$-weighted anatomical images using a linear image registration tool (flirt, FSL, RRID: SCR_002823), and then displayed as statistical $t$ value maps. Probability activation maps were generated from $t$ value maps (FDR-corrected, with a threshold of $t$ value $=2, p<0.05)$ of all runs obtained within the same imaging session (e.g., see Figs. $2 A, 4 A$ ). We quantified the activation detection rate (activated runs/the total number of runs) for both areas $3 \mathrm{~b}$ and $\mathrm{S} 2$ in pre- and post-SCI conditions. BOLD signal time courses were extracted from a single peak voxel with the highest probability of activation in areas $3 \mathrm{~b}$ and S2 for quantifying amplitudes of BOLD responses to stimuli. The peak responses of areas $3 \mathrm{~b}$ and $\mathrm{S} 2$ were compared before and after the DCL.

The voxels exhibiting the highest activation probability were selected as the seeds in areas $3 \mathrm{~b}$ and S2 for rsfMRI signal analyses. Digit representation maps identified with electrophysiology were also used to adjust the selection of seeds. Resting-state BOLD signals extracted from each seed were then used to perform voxelwise and seed pairwise correlation analyses. Seed-based voxelwise correlation coefficients map (3dfim, AFNI) was generated to reveal the overall FC pattern of each seed in areas $3 \mathrm{~b}$ and S2. Correlation maps were thresholded (e.g., see Fig. $4 D, E$, $r$ value $=0.6$ on S1 maps and $r$ value $=0.5$ on S2 maps), spatially interpolated, and overlaid on anatomical images for display, with warm color indicating high correlation value. The pairwise correlation values between area $3 \mathrm{~b}$ and $\mathrm{S} 2$ seeds were calculated and compared in pre- versus post-SCI conditions (1dCorrelate, AFNI). Both surface and transcortical blood vessel features were prevalent and easily identifiable on anatomical MRI images; therefore, these land markers were used for coregistering MRI maps obtained in different imaging sessions to surface blood vessel maps obtained later during microelectrode mapping/recording sessions in each animal (Fig. 2).

Identification of area $3 b$ and $S 2$ regions and extracellular recording. In each animal, fMRI activation maps were used as guides for microelectrode penetrations placed around the central sulcus and into the upper bank of the lateral sulcus, where digit regions in areas $3 \mathrm{~b}$ and $\mathrm{S} 2$ reside. For mapping purposes, single epoxylite-coated tungsten microelectrode with an exposed standard sharp tip $(<3 \mu \mathrm{m})$ of $\sim 1 \mathrm{~m} \Omega$ impedance (measured at $1 \mathrm{kHz}, \mathrm{FHC}$ ) was inserted through cortical depth of area $3 \mathrm{~b}$ digit regions $(0-1500 \mu \mathrm{m}$ in depth). At the face area $3 \mathrm{~b}$ region, which is located lateral to the digit regions, microelectrode was further advanced (3500-5000 $\mu \mathrm{m}$ in depth) into the upper bank of the lateral sulcus to localize S2 digit region. The depth of the electrode tip was tracked and stopped at $300 \mu \mathrm{m}$ intervals. At each stop, the receptive field of neurons (e.g., digit or face) were characterized and logged. Neuronal response magnitudes to digit or face stroking or tapping with cotton swab were quantified on a six-level scale (i.e., no, very weak, weak, good, very good, or excellent response). After the completion of the mapping procedure, we were able to identify area $3 \mathrm{~b}, \mathrm{~S} 2$, and interareal borders based on the known characteristics of receptive field properties, preferred stimuli, and somatotopic organization of the digits, hand, and arm.

For recordings, a pair of electrodes were placed in area 3b and S2 digit regions showing very good or excellent response for two-site simultaneous recordings. Electrodes were advanced separately into cortex using a hydraulic microdrive. To drive both area $3 \mathrm{~b}$ and S2 neurons simultaneously and effectively, tactile stimuli were presented at their shared receptive field (e.g., digit 2 tip). The recording sites in area $3 \mathrm{~b}(700-900 \mu \mathrm{m}$ in depth) and S2 (3500-5000 $\mu \mathrm{m}$ in depth) of each animal are shown in Figures 1 and 2 (black arrows). Spiking and LFP activities were recorded simultaneously with a Multichannel Acquisition Processor System (Plexon) and outputted into two separated channels after undergoing 
A

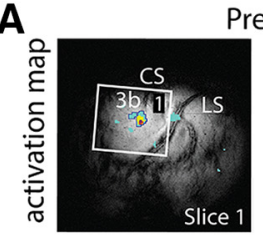

B

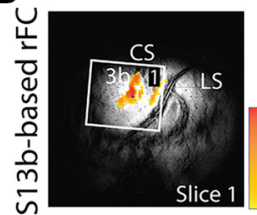

Pre-lesion
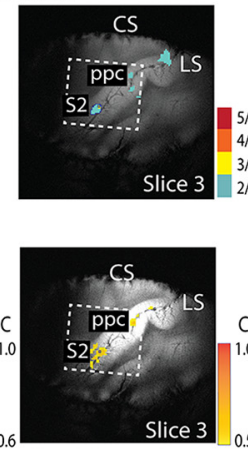

C

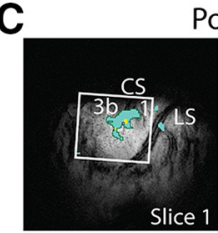

D

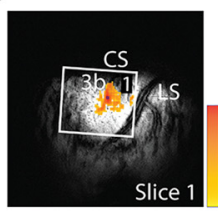

Post-lesion
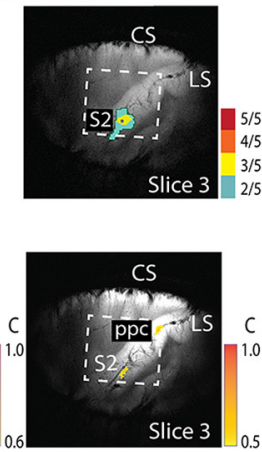

E

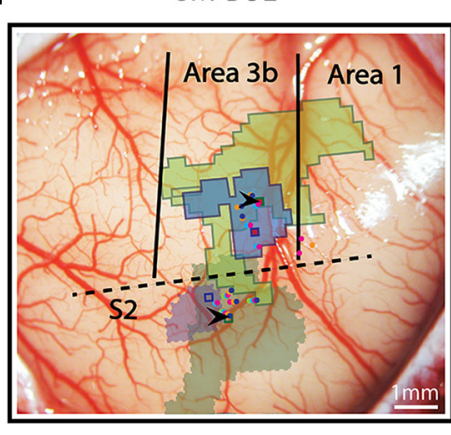

$\stackrel{M}{\perp}$

acti_PreL_S13b acti_PreL_S2 acti_PostL_S13b acti_PostL_S2 Seed: PreL_S13b - Seed: PreL S2 Seed: PostL_S13b Seed: PostL_S2

D1 mapping

D2 mapping

- D3 mapping

D4 mapping

- D5 mapping
$F$

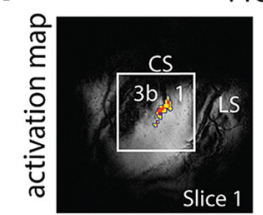

G

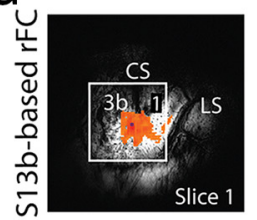

Pre-lesion
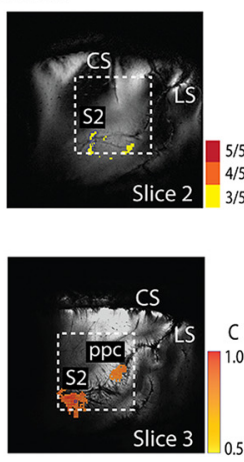

H

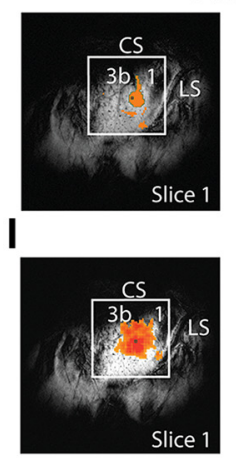

Post-lesion
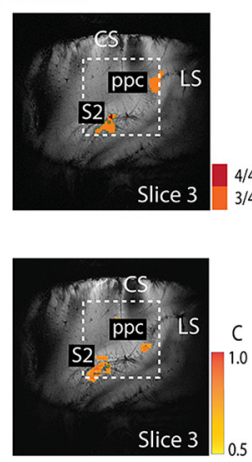

J

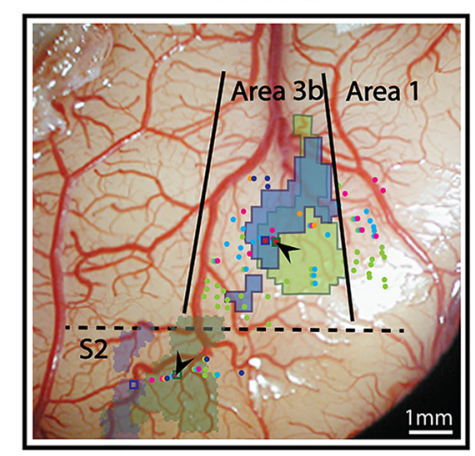

$\stackrel{M}{+}$ acti_PreL_S13b acti_PreL_S2 acti_PostL_S13b acti_PostL_S2 - Seed: PreL_S13b Seed:PreL_S2 Seed: PostL_S13b Seed: PostL_S2

- D1 mapping

- D2 mapping

- D3 mapping

D4 mapping

- D5 mapping

> Ele_site

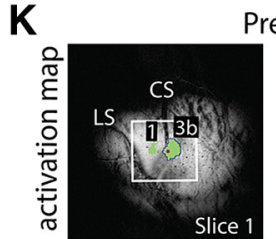

L

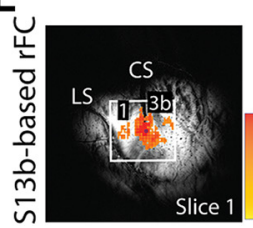

Pre-lesion
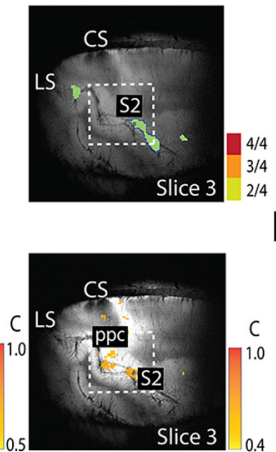

M

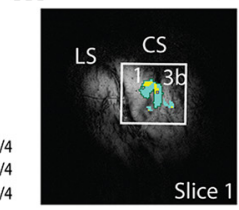

N

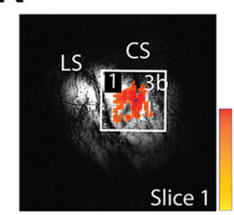

Post-lesion
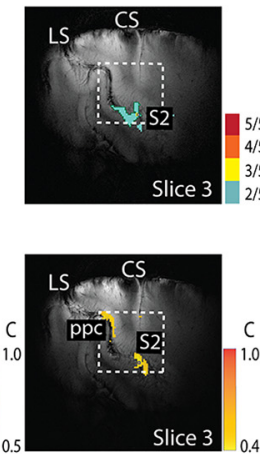

0

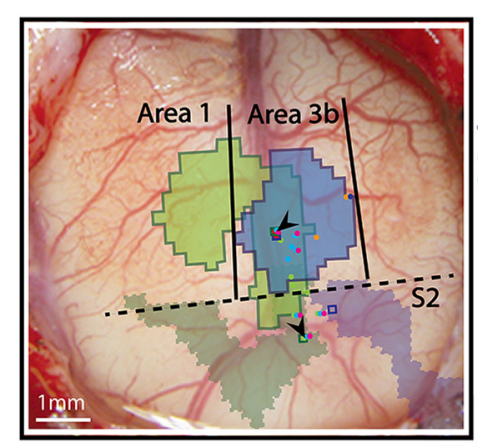

$P+A$

- acti_PreL_S13b acti_PreL_S2 9. acti_PostL_S13b acti_PostL_S2 - Seed: PreL_S13b - Seed: PreL_S2 - Seed: PostL_S13b - D1 mapping - D2 mapping - D3 mapping - D4 mapping - D5 mapping

Figure 2. Comparison of the stimulus-evoked fMRI activation and voxelwise rsFC maps of areas 3 b and S2 before and after DCL conditions in 3 animals: SM-BUL ( $\boldsymbol{A}-\boldsymbol{E})$, SM-GUA ( $\boldsymbol{F}-\boldsymbol{J})$, and SM-SUP $(\boldsymbol{K}-\mathbf{0}) . \boldsymbol{A}, \boldsymbol{C}, \boldsymbol{F}, \boldsymbol{H}, \boldsymbol{K}, \boldsymbol{M}$, Probability activation maps to single digit tactile stimulation in pre-DCL $(\boldsymbol{A}, \boldsymbol{F}, \boldsymbol{K})$ and post-DCL $(\boldsymbol{C}, \boldsymbol{H}, \boldsymbol{M})$ conditions. In the imaging session shown in $\boldsymbol{F}$, it was determined that S2 cortex was sampled on slice 2 based on anatomical landmarks. $\boldsymbol{B}, \boldsymbol{D}, \mathbf{G}, \boldsymbol{I}, \boldsymbol{L}, \boldsymbol{N}$, rsFC maps of area 3 b seeds in pre-DCL $(\boldsymbol{B}, \boldsymbol{G}, \boldsymbol{L})$ and post-DCL $(\boldsymbol{D}, \boldsymbol{I}, \boldsymbol{N})$ conditions. $\boldsymbol{E}, \boldsymbol{J}, \mathbf{O}, 0$ verlaid pre- and post-DCL

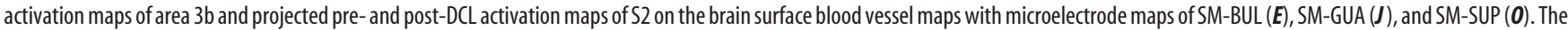
paired area 3b-S2 recording sites, indicated by black arrows, are responsive to the same digit stimulation (i.e., sites share receptive fields). Small boxes represent resting-state seeds: blue boxes represent prelesion; green boxes represent postlesion. White squares on MRI images represent the FOV of the blood vessel maps. Blue and green outlines represent prelesion and postlesion activation patterns, respectively. Solid and dashed outlines indicate the area $3 \mathrm{~b}$ and $\mathrm{S} 2$ activation maps. Colored dots indicate the microelectrode mapping sites, with different colors representing receptive fields of different digits ( $\boldsymbol{E}, \boldsymbol{J}, \mathbf{0}$, right, column insets) $A$, Anterior; $L$, lateral; $M$, medial; $P$, posterior.

different analog filtering. The electrical signals on each channel were amplified 5000 times and band-filtered between $300 \mathrm{~Hz}$ and $8 \mathrm{kHz}$. Spiking activity and LFP signals were sampled at $30 \mathrm{kHz}$ and $2 \mathrm{kHz}$ rates, respectively. Representative microelectrode mapping and recording maps are shown in Figure $1 D-F$.

Extracellular recording and analysis. We identified area 3b, S2, and interareal borders by using fMRI map, electrode mapping, and vasculature pattern as guides, and based on the known characteristics of receptive field properties, preferred stimuli, and somatotopic organization of the digits, hand, and arm (Kaas et al., 1984; Jain et al., 2008; Wang et al., 2013). Detailed surgical procedures were described previously (Chen et al., 2001; Reed et al., 2008; Wang et al., 2013).

We used custom-written codes in MATLAB (The MathWorks, RRID: SCR_001622) for electrophysiological data processing and statistical analysis. Spike sorting, peristimulus time histogram (PSTH), and joint PSTH (JPSTH) analyses were done in NeuroExplorer software (Plexon, RRID: SCR_001818). Raw LFP signals were first filtered with a $60 \mathrm{~Hz}$ and $120 \mathrm{~Hz}$ band stop Chebyshev filter and then were bandpass filtered between $1 \mathrm{~Hz}$ and $150 \mathrm{~Hz}$. Event-related LFP signal changes were averaged from 100 trails obtained within each run for display (see Fig. $5 A, B$ ). The power spectrums of LFP signals at resting and stimulation states were calculated. Magnitude squared coherence between areas 3b and S2 at resting state (with $4 \mathrm{~Hz}$ frequency and $0.5 \mathrm{~s}$ temporal resolutions) was estimated. Broadband LFP signals were divided into seven frequency bands for quantification: delta $(1-4 \mathrm{~Hz})$, theta $(5-8 \mathrm{~Hz})$, alpha $(9-12 \mathrm{~Hz})$, beta $(13-30 \mathrm{~Hz})$, low gamma (1_gamma, 30-50 Hz), high gamma (h_gamma, 50-100 Hz), and very high gamma bands (vl_gamma, 100$150 \mathrm{~Hz}$ ). Percentages of power change at all LFP bands were calculated by 
subtracting and then dividing their corresponding baseline power at resting state.

Multiunit spikes were used in response magnitude quantification and synchrony analysis. Single neuron spiking activity was first sorted and then combined together as multiunits for further analysis (Rasputin software, Plexon). PSTHs of spikes of area $3 \mathrm{~b}$ and $\mathrm{S} 2$ neurons were calculated to determine the firing rates (see Fig. $6 A, B$ ). JPSTH between area $3 \mathrm{~b}$ and S2 neurons were computed to investigate dynamic firing synchrony (see Fig. 6C). We used $10 \mathrm{~ms}$ as the bin width with reference to previous studies under similar experimental conditions (Reed et al., 2008, 2012; Wang et al., 2013). Percentages of spiking rate changes were calculated by subtracting and then dividing the baseline spiking rate at resting state. Spiking synchrony between area $3 \mathrm{~b}$ and S2 neurons was measured with the cross-correlation histogram using NeuroExplorer software (Plexon). The peak correlation value from the cross-correlation histogram was compared between the normal and lesioned animals.

SCI and histological evaluation of the level and extent of DCL. Unilateral dorsal column section of the cervical spinal cord was performed under surgical-level anesthesia (1.5\%-3\% isoflurane). Under aseptic conditions, a portion of the cervical spinal cord at C4-C6 level was exposed, and the dorsal columns were sectioned on one side at C5 level with a pair of fine surgical scissors. The dura was replaced with Gelfilm and covered with Gelfoam.

To determine at which cervical segment level the section was placed and to what extent the dorsal column afferents were disrupted, we injected cholera toxin B subunit (CTB, $5 \mu \mathrm{m}$ per site) tracer into the finger pads of D1, D3, and D5 of both hands and performed thorough gross examination of the postmortem spinal cord as well as microscopic examination of sectioned and stained spinal cord tissue. Several procedures were performed to ensure an accurate determination of cervical segments. First, we exposed the entire spinal cord and all cervical afferent bundles on postmortem body. This permits the identification of the injury site and its location on segment clearly. Second, we placed pin markers at the middle of dorsal afferent entering zone on several segments, and these pin holes are visible on coronal spinal cord sections (see Fig. 8G, two holes at C3 and C6 level in SM-SUP). We routinely dissect the cord at $\mathrm{C} 2$ and $\mathrm{C} 8$ levels for histology. Third, using the CTB terminals on the normal side of the spinal cord as segmental references, we determined at which digit level the lesion was placed. Fourth, similarly, by comparing the sizes of CTB-stained regions and their somatotopic arrangements in the cuneate nuclei of the brainstem, and considering the normal intact side as $100 \%$, we confirmed the injury level (e.g., at which digits based on the stain pattern) and quantified the percentage of afferent disruption for each animal. Together, all (or part) of above mentioned assessments enabled us to accurately confirm the level of injury and quantify the extents of afferent disruption. Detailed histology data from these 3 monkeys are shown in Figure 8. In 2 cases, the CTB stains were not apparent (8D and $8 \mathrm{G}$ ), but gross anatomy and CTB stains at cuneate nuclei confirmed the lesion was placed at D1 level in these 2 animals, respectively. Additional details about surgical, postsurgical procedures, and quantification method can be found in our previous publications (Qi et al., 2011; Chen et al., 2012; Wu et al., 2016).

The hand use behavior of the animal was evaluated also from the following two aspects: home-cage activity and food reaching-graspingretrieving behavior. After unilateral DCL, the affected hands were hardly used for any in-cage activity, such as food reaching and climbing, for the first $3 \mathrm{~d}$ immediately after lesion. Within the first 2 postlesion weeks, the hand posture and uses were abnormal. We often observed that animals tended not to use their impaired hand but effectively did so when forced to use their affected hand to climb cages or grasp and hold food. No other apparent motor deficit was noticed. Animals were also trained in their home cage on food reaching/grasping/retrieving tasks before DCL. The task involved reaching and retrieving a small sugar pellet from a well on a modified Kluver board $(15.2 \times 9.9 \mathrm{~cm})$. Four wells (wells $1-4)$ with different depths $(0.14,0.28,0.38$, and $0.64 \mathrm{~cm}$, respectively) and diameters $(0.78,1.20,1.14$, and $1.14 \mathrm{~cm}$, respectively) were created to introduce incremental difficulties into the task, with well 1 being the easiest and well 4 being the hardest. Within each behavioral session, the performances of an animal on 80 task trials (20 trials for each well) were recorded using a
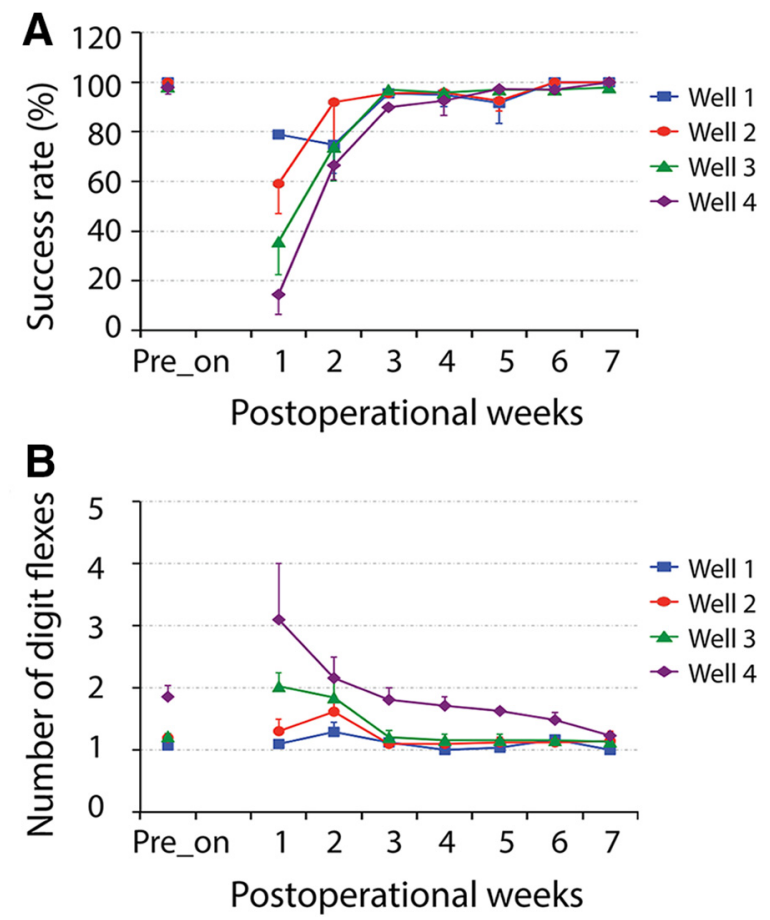

Figure 3. Longitudinal evaluation of food-reaching and food-grasping behaviors following cervical DCL. $\boldsymbol{A}, \boldsymbol{B}$, Plots of success rates $(\boldsymbol{A})$ and number of digit flexes $(\boldsymbol{B})$ at different postoperational time points (weeks) for SM-SUP. Error bars indicate SE.

high-speed (60 frames/s) video camera. Behavioral data were collected 3 times before the lesion (one mean value generated) and then daily after the lesion (after skipping the first $3 \mathrm{~d}$ immediately after lesion). Daily sessions were summed to generate a single performance score for each week. The procedures were similar to those detailed in previous reports (Wu et al., 2016). A successful food retrieval rate and the number of digit flexes were measured for quantitative scoring. A single flexion of the digits for a successfully retrieved pellet was considered to be a perfect score. The success rate (Fig. $3 A$ ) and number of digit flexes (Fig. $3 B$ ) dropped for 2 weeks after the lesion and then returned to prelesion level at $\sim 4$ weeks after lesion in the representative case (SM-SUP).

Experimental design and statistical analysis. $\mathrm{fMRI}$ data of the 3 monkeys that underwent scans before and after SCI were included in the group analysis, including 21 prelesion and 14 postlesion runs for stimulation condition, and 23 prelesion and 13 postlesion runs for resting-state condition. LFP and spiking data were collected from all 6 monkeys. Paired simultaneous S2 and area $3 \mathrm{~b}$ electrophysiological recording data from a total of 32 runs ( 20 runs from normal and 12 runs from lesioned animals) were quantified and compared. Unpaired $t$ test was performed to determine whether the stimulus-evoked fMRI and LFP/spike signal changes in areas $3 \mathrm{~b}$ and $\mathrm{S} 2$ and resting-state connectivity strengths differed significantly between normal and lesion conditions. Percentage of stimulusevoked signal change and rsFC change caused by deafferentation were obtained by subtracting, and then dividing the signals in postlesion condition $\left.\left(\mathrm{dS} / \mathrm{S}=\mathrm{S}_{- \text {postlesion }}-\mathrm{S}_{- \text {prelesion }}\right) / \mathrm{S}_{- \text {prelesion }}\right)$. Pearson correlation analysis was performed to evaluate the relationship between the overall trends (curves) of stimulus-evoked signal and rsFC changes induced by deafferentation. A $p$ value $<0.05$ was considered statistically significant, and results were expressed as mean $\pm \mathrm{SE}$.

\section{Results}

\section{DCL reduces stimulus-evoked fMRI responses and rsFC}

In normal prelesion cortex, tactile stimulation of a single digit evoked robust and reproducible fMRI activations in several cortical areas, including areas $3 \mathrm{~b}$ and S2 (Fig. 4A). After DCL, fMRI activations were relatively less stable with altered spatial activation patterns in input-deprived areas $3 \mathrm{~b}$ and S2 (Fig. 4B). Direct 
A

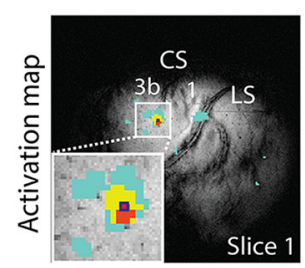

D

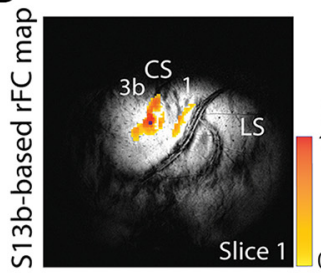

Pre-lesion
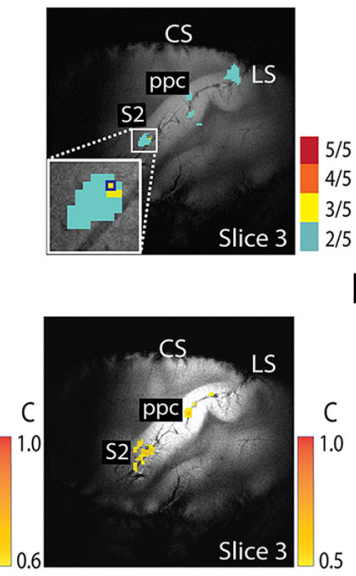

B

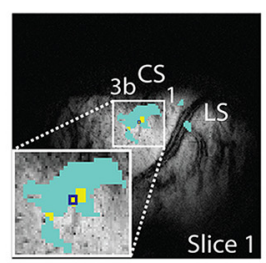

E

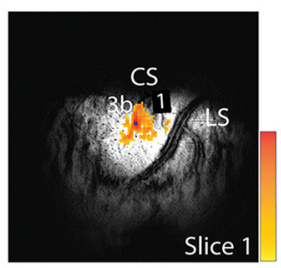

Post-lesion
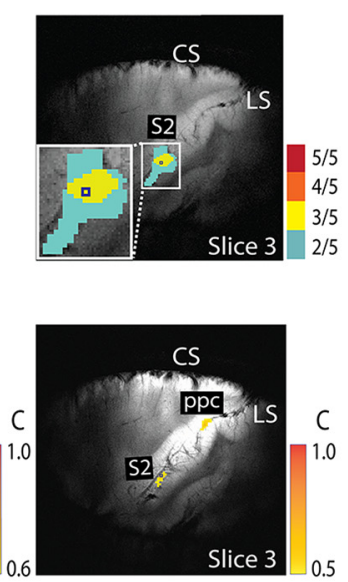

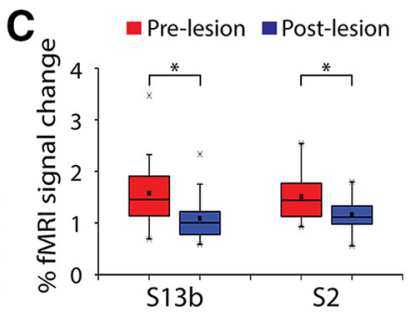

$\mathbf{F}$

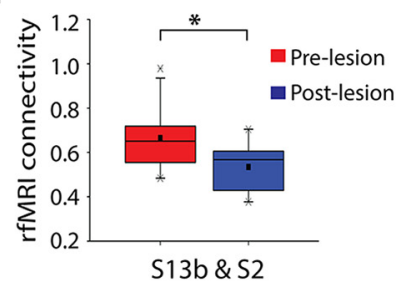

Figure 4. Effects of DCL on tactile stimulus-evoked fMRI response and rsFC between areas 3b and S2. A-C, The activation probability maps in S1 (left image) and S2 (right image) to tactile stimulation on D2/D3 in the prelesion normal $(\boldsymbol{A})$ and postlesion deafferented $(\boldsymbol{B})$ cortex. $5 / 5$ indicates 5 of 5 runs. Small blue squares represent the seed voxels. $\boldsymbol{C}$, Group mean percentage $\mathrm{fMRI}$ signal change to stimuli in area 3b (left columns) and S2 (right columns) pre-DCL (red columns) and post-DCL (blue columns) DCL. D-F, Corresponding rsFC maps of the area 3b (left) and S2 (right) seeds in prelesion normal $(\boldsymbol{D})$ and postlesion deafferented $(\boldsymbol{E})$ conditions. $\boldsymbol{F}$, Group mean correlation coefficient values between areas $3 \mathrm{~b}$ and S2 pre-DCL (red column) versus post-DCL (blue column). A total of 21 prelesion and 14 postlesion runs from 3 animals were acquired for $\mathrm{FRRI}$ response measurement ( $\mathrm{df}=33$ ). A total of 23 prelesion and 13 postlesion runs from 3 animals were acquired for rsfMRI $\mathrm{FC}$ measurement ( $\mathrm{df}=34) .{ }^{*} p<0.05$ (unpaired $t$ test). Error bars indicate SE. The representative case is SM-BUL in $\boldsymbol{A}, \boldsymbol{B}$ and $\boldsymbol{D}, \boldsymbol{E}$.

comparisons of prelesion and postlesion fMRI activation maps clearly revealed these alterations (for details, see Fig. 2). The group mean magnitudes of fMRI signal change in response to identical stimuli were significantly reduced by $\sim 25 \%$ in both areas (area 3b: $1.6 \pm 0.1 \%$ prelesion, $1.1 \pm 0.1 \%$ postlesion, unpaired $t$ test, $p=0.01$; S2: $1.5 \pm 0.1 \%$ prelesion, $1.1 \pm 0.1 \%$ postlesion, unpaired $t$ test, $p=0.017$; Fig. $4 C$ ). Using the voxels with the most reproducible (i.e., the highest probable) activation as seeds, we found that the postlesion local and interareal rsFC patterns of areas $3 \mathrm{~b}$ and S2 differed markedly (compare Fig. $4 E$ with Fig. 4D). The group pairwise mean rsFC between areas $3 \mathrm{~b}$ and $\mathrm{S} 2$ reduced $\sim 20 \%$ after lesion (correlation coefficient reduced from $0.66 \pm 0.03$ to $0.53 \pm 0.03$, unpaired $t$ test, $p=0.005$; Fig. $4 F)$.

\section{DCL reduces LFP responses to stimuli and interareal coherences}

Event-averaged LFP time courses showed robust LFP amplitude increases at the stimulus onset and offset in normal areas $3 \mathrm{~b}$ and S2 (Fig. 5A). The response amplitudes in input-deprived cortex were much weaker than those in normal cortex; signal was almost abolished during the stimulus offset period (Fig. $5 B$ ). To understand the consequences of input deprivation on stimulus-evoked responses and rsFC, we compared the group mean LFP power at seven frequency bands in four condition pairs: stimulus-evoked LFP in normal versus lesion, resting-state LFP in normal versus lesion, stimulus-evoked LFP versus resting state in normal condition, and stimulus-evoked LFP versus resting state in lesioned condition (Fig. 5D,E). In normal area $3 \mathrm{~b}$ and $\mathrm{S} 2$ cortices, the stimulus-evoked LFP power increases in all frequency bands (Fig. $5 D, E$, compare red and pink columns). Although statistically significant increases were detected in all frequency bands in S2, only beta, all gamma, and broad bands showed significant increases in area $3 b$. After DCL, the stimulus-evoked LFP power was reduced in all frequency bands, but with significant reductions in beta and all three gamma bands for area 3 b (Fig. $5 D$, compare pink with light blue columns), and in just the three gamma bands for S2 (Fig. 5E). At resting state, the LFP power was generally higher in low-frequency bands, but the power differ- ences between stimulation and resting states were more prominent in low and high gamma bands in both areas $3 \mathrm{~b}$ and S2. After lesion, these power differences between stimulation and resting states were drastically reduced (Fig. 5D,E, blue and light blue columns). Statistically significant differences were present only in the three gamma bands in both areas $3 \mathrm{~b}$ and S2 (Fig. 5D, E, compare blue and light blue columns). Significant power differences between normal and lesion conditions during resting state were present only in the very high gamma band in area $3 \mathrm{~b}$ and in the high gamma band in S2 (Fig. 5D,E, compare red with blue columns). Similar patterns were also observed in low LFP frequency bands, but no statistical significance was found. In summary, the most drastic lesion-induced LFP power changes were observed in high-frequency bands of LFP signals in both areas $3 \mathrm{~b}$ and S2. These changes occurred in both stimulation and resting states.

We next examined how input deprivation affects LFP rsFC, as measured by magnitude-squared coherence, between areas $3 \mathrm{~b}$ and S2. Strong FC occurred in the low-frequency range of LFP (i.e., delta, theta, and alpha bands) in both normal and inputdeprived cortex (Fig. 5C,F). After DCL, the coherence was weakened significantly across most LFP frequency bands, except the delta band (Fig. 5F).

\section{Lesion reduces the spike activities of area $3 b$ and S2 neurons and disrupts their synchrony}

In normal areas $3 \mathrm{~b}$ and S2, PSTH plots of multiple-unit spiking rates showed robust and transient increases during the stimulus onset/offset periods with a moderate increase in firing rate during the sustained stimulus period (Fig. 6A). Spiking rate reduced in both areas after DCL, most apparent during the stimulus offset and sustained stimulus periods (Fig. 6B). In input-deprived areas $3 \mathrm{~b}$ and $\mathrm{S} 2$, the stimulus-evoked spiking activities were significantly reduced (Fig. 6E, F, compare light red and light blue columns). The main JPSTH matrix plots in Figure $6 \mathrm{C}$ showed dynamic firing synchrony of S2 and area $3 \mathrm{~b}$ neurons under normal and lesioned conditions. We examined the rsFC of area $3 \mathrm{~b}$ and S2 neurons by calculating the cross-correlation of their spontaneous spike activity in normal and input-deprived cortex. One representative cross-correlation histogram shows that the syn- 

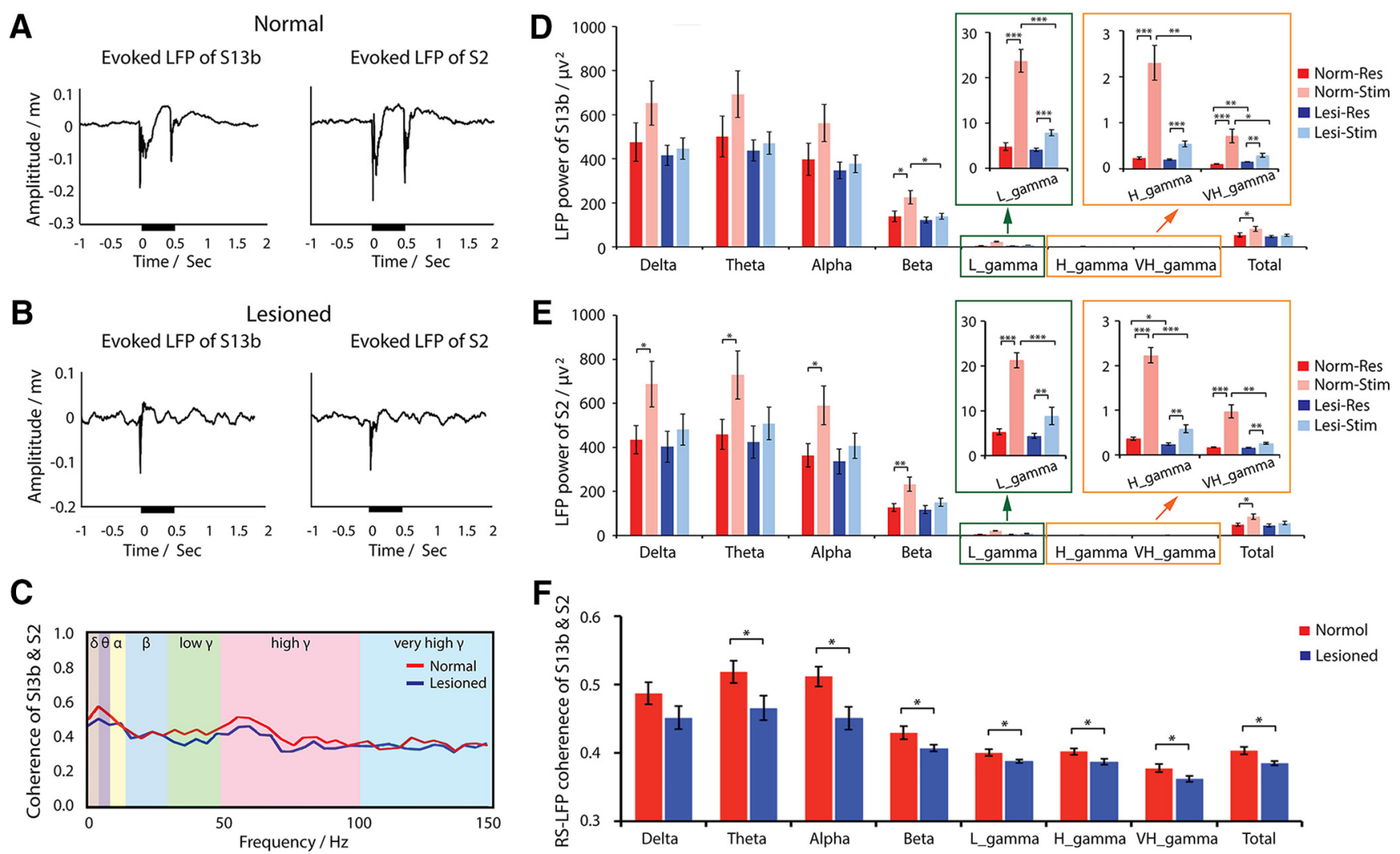

Figure 5. Effects of DCL on tactile stimulus-evoked LFP signal and resting-state LFP coherence between area $3 \mathrm{~b}$ and $S 2$ neurons. $\boldsymbol{A}, \boldsymbol{B}$, Examples of event-averaged LFP signal amplitude to stimuli in areas $3 \mathrm{~b}$ (left) and $\mathbf{S 2}$ (right) in normal $(\boldsymbol{A})$ and lesioned $(\boldsymbol{B})$ conditions. Black lines near the $x$-axes indicate the stimulus duration. $\boldsymbol{C}$, Example of LFP coherence Spectrum between areas $3 \mathrm{~b}$ and $\mathbf{S 2}$ at resting state in normal (red line) and lesioned (blue line) animals. Different background colors represent different frequency bands. $\boldsymbol{D}, \boldsymbol{E}$, Group comparison of LFP power of different frequency bands in areas $3 \mathrm{~b}(\boldsymbol{D})$ and $\mathbf{S 2}(\boldsymbol{E})$ in stimulation and resting states in normal and lesioned animals. $\boldsymbol{F}$, Group LFP coherence between areas $3 \mathrm{~b}$ and $\mathrm{S} 2$ at resting state in normal (red columns) versus deafferented (blue columns) conditions. Paired simultaneous $\$ 2$ and area 3 b electrophysiological data from 20 runs from 3 normal animals, and 12 runs from 3 lesioned animals were included (df = 30). Unpaired $t$ test between normal and deafferented groups, paired $t$ test within each group: ${ }^{*} p<0.05 ;{ }^{* *} p<0.005$; ${ }^{* * *} p<0.0005$. Error bars indicate SE.

chrony is altered in two ways after lesion (Fig. 6D): the strength was markedly reduced, and the temporal delay (lag) between areas $3 \mathrm{~b}$ and S2 present in normal cortex disappeared (Fig. 6D, comparing red and blue lines). The reduction in cross-correlation strength between spontaneous spiking of neurons in input-deprived areas $3 \mathrm{~b}$ and $\mathrm{S} 2$ was significant at the group level (unpaired $t$ test, $p=0.002$; Fig. $6 G)$.

Interareal differences in stimulus responsiveness reductions induced by input deprivation across different signal types

While the DCL universally reduced cortical responsiveness to stimuli across all three types of signals, the most apparent interareal differences between areas $3 \mathrm{~b}$ and S2 were observed in LFP, particularly in the low-frequency range (Fig. $7 A-D)$. For example, in normal cortex, the largest interareal differences in response to tactile stimuli were detected in gamma range LFP signals, with low-gamma LFP signal showing statistical significance (Fig. 7C). The overall responsiveness to stimuli dropped drastically across all signals in both areas (Fig. $7 A, B$ ) weeks after DCL, the most pronounced interareal differences were observed in low-frequency range bands of LFP signals (Fig. 7D, delta, theta, and alpha bands; see Fig. $7 C, D$, the $y$-axis scale bar differences).

Comparisons of DCL effects on the relationships between stimulus responsiveness and rsFC across fMRI, LFP, and spiking signals

We last examined how alterations in stimulus responsiveness induced by input deprivation in areas $3 \mathrm{~b}$ and $\mathrm{S} 2$ relate to their interareal rsFC changes, and how the alterations in the three types of signals compare with each other. Compared with normal cortex, FC drastically weakened across all signals in the inputdeprived cortex (Fig. 7E, compare red and blue columns). In general, the response reductions were much greater than the rsFC strength changes (Fig. 7F, compare red and blue lines with green line) across all signal types, but especially for the LFP signal. The most robust rsFC weakening induced by DCL was observed in the spike signal with a $31 \%$ decrease, compared with $19 \%$ in fMRI and $~ 10 \%$ (7.3\% in delta, $10.1 \%$ in theta, $11.9 \%$ in alpha, $6.0 \%$ in beta, $3.6 \%$ in low gamma, $3.8 \%$ in high gamma, and $4.5 \%$ in very high gamma bands) in LFP (Fig. $7 F$, green line). The most robust reductions in stimulus-evoked signals were found in LFP for both areas $3 \mathrm{~b}$ and $\mathrm{S} 2$, with $\sim 80 \%$ descend (area $3 \mathrm{~b}: 91.6 \%$ in delta, $91.5 \%$ in theta, $91.2 \%$ in alpha, $90.1 \%$ in beta, $88.8 \%$ in low gamma, $85.5 \%$ in high gamma, $86.4 \%$ in very high gamma bands; S2: $76.2 \%$ in delta, $76.1 \%$ in theta, $76.0 \%$ in alpha, $76.1 \%$ in beta, $82.8 \%$ in low gamma, $84.6 \%$ in high gamma, $88.0 \%$ in very high gamma bands), compared with $\sim 25 \%$ in fMRI (area $3 \mathrm{~b}: 31 \%$; S2: $22 \%$ ) and $\sim 70 \%$ in spike (area 3b: $68.8 \%$; S2: 74.1\%). The largest reduction difference $(>65 \%)$ was observed in LFP signals across all frequency bands, which showed $\sim 10 \%$ reduction in rsFC but $>75 \%$ decrease in response magnitudes to stimuli (Fig. $7 F$, blue column). The differences in spiking were $\sim 40 \%$ (Fig. $7 F$, yellow column) and $\sim 10 \%$ for $\mathrm{fMRI}$ (Fig. $7 F$, pink column). To understand the relationship between the reductions in stimulus responsiveness and FC weakening across all three types of signals in areas $3 \mathrm{~b}$ and $\mathrm{S} 2$, we performed correlation analysis of the 
A

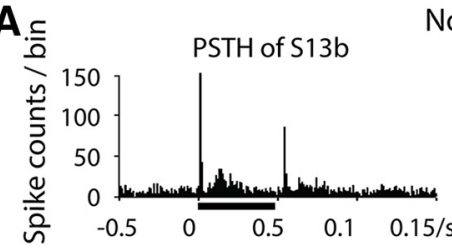

Normal

B.

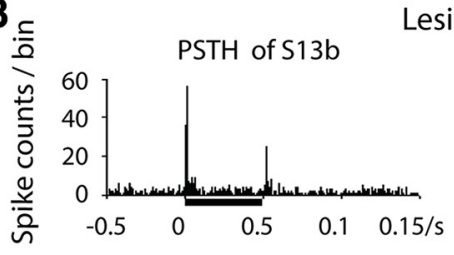

Lesioned

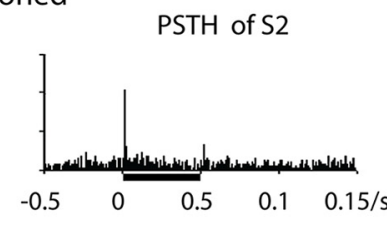

C

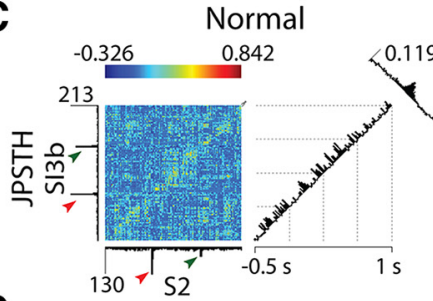

D.

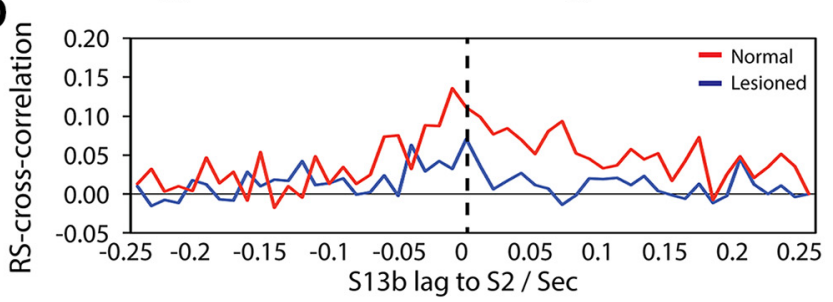

E

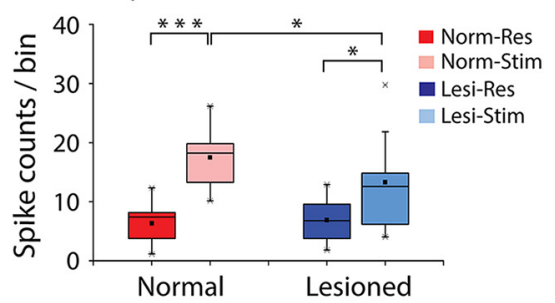

$\mathbf{F}$

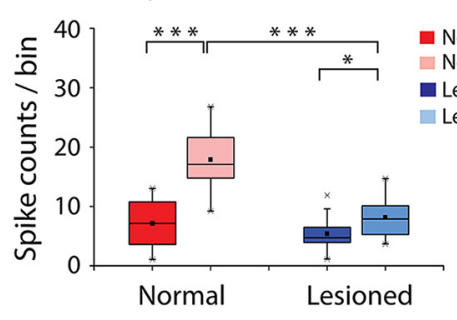

G

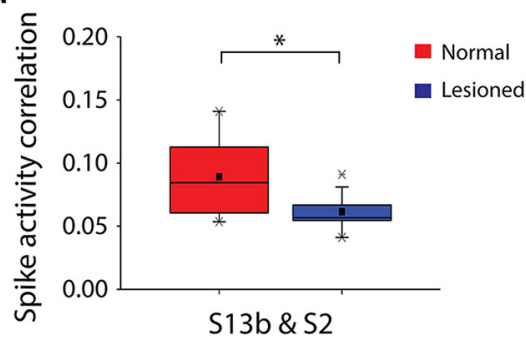

Figure 6. Effects of DCL on tactile stimulus-evoked spike activity and cross-correlation of spontaneous resting-state spike activity between area $3 \mathrm{~b}$ and $\mathrm{S} 2 \mathrm{neurons}$. $\boldsymbol{A}, \boldsymbol{B}$, Examples of PSTHs in area $3 \mathrm{~b}$ (left) and S2 (right) in normal $(\boldsymbol{A})$ and deafferented $(\boldsymbol{B})$ conditions. Black bars next to $x$-axes represent tactile stimulation duration. Time of bin $=10 \mathrm{~ms}$. $\boldsymbol{C}$, Examples of JPSTH of areas $3 \mathrm{~b}$ and S2 in normal (left) and lesioned (right) monkeys. The main JPSTH matrix plot represents the correlation of bin (bin $=10 \mathrm{~ms}$ ) counts of $\$ 2$ and area $3 \mathrm{~b}$ neurons around the vibrotactile stimulation event: red arrows indicate stimulus onset; green arrows indicate stimulus offset. Histograms to the left of (area 3b) and below (S2) the matrix are standard perievent histograms. The first (diagonal) histogram to the right of the matrix represents the correlations of near-coincident spikes around the stimulus events. The far-right (oblique) histogram represents the cross-correlations of firings of area $3 \mathrm{~b}$ and $\mathrm{S} 2$ neurons. The peak firing counts and peak correlations are labeled. For example, in normal monkey (left), the peak firing counts of area $3 \mathrm{~b}$ is 213 and the peak firing counts of 52 is 130 , whereas the peak spike activity correlation of areas $3 \mathrm{~b}$ and $S 2$ is 0.119 . D. Examples of cross-correlation of spike activities between area $3 \mathrm{~b}$ and $\mathrm{S} 2$ neurons at resting state in normal (red line) and deafferented (blue line) cortex. $\boldsymbol{E}, \boldsymbol{F}$, Group comparison of spike rates in stimulation versus resting states in area $3 \mathrm{~b}(\boldsymbol{E})$ and $\mathrm{S2}(\boldsymbol{F})$ in normal versus deafferented cortex. Red columns represent resting state in normal animal. Pink columns represent stimulation in normal animal. Blue columns represent resting state in lesioned animal. Light blue columns represent stimulation in lesioned animal. $G$, Comparison of spike cross-correlation between area $3 \mathrm{~b}$ and $\mathrm{S} 2$ neurons at resting state in normal (red columns) versus input-deprived (blue columns) animals. A total of 20 runs from 3 normal animals, and a total of 13 runs from 3 lesioned animals were acquired for spike measurement ( $\mathrm{df}=31$ ). ${ }^{*} p<0.05$ (unpaired $t$ test). ${ }^{* * *} p$ value $<0.0005$ (unpaired $t$ test). Error bars indicate SE.

deafferentation-induced signal reduction curves with the rsFC strength reduction curve. This revealed strong and positive correlations between the stimulus-evoked response reduction in areas $3 \mathrm{~b}$ and S2 $(r=0.91, p=0.0002$; Fig. $7 F$, comparing red and blue lines), but negative yet insignificant correlations between the rsFC strength reduction and stimulus-evoked signal reduction in both area $3 \mathrm{~b}(r=-0.60, p=0.06$; Fig. $7 F$, comparing green and blue lines) and S2 $(r=-0.47, p=0.17$; Fig. $7 F$, comparing green and red lines).

\section{Histological evaluation of the extent and spinal cord levels of} the unilateral DCL

Postmortem histology was used to evaluate the percentage of afferent disruption after DCL, as well as the level and extent of the SCI (Fig. 8). Using the pin markers and appearance of CTB terminal patches of three digits on the intact side of the spinal cord as references, the levels of lesion tissue destruction were determined to be in D1 for all 3 monkeys (Fig. 8A,D,G). CTBimmunoreacted axial sections of the brainstem showed that the CTB staining on the intact side was robust and well organized, but that on the lesioned side was not (Fig. $8 B, E, H$ ), indicating that the lesions were extensive. Further, the percentage of afferent disruption of each monkey was quantified. The mean afferent disruption was 97\% (97\% for SM-BUL, 98\% for SM-GUA, and $96 \%$ for SM-SUP, respectively). Additionally, as shown in reconstructed transverse views of the spinal cord, the lesion (Fig. $8 C, F, I$, black shaded areas) successfully disrupted the dorsal column.

\section{Discussion}

Input deprivation, responses to peripheral tactile stimuli, and interareal FC between areas $3 \mathrm{~b}$ and $\mathrm{S} 2$

Many neurological disorders, such as stroke, spinal or brain injury, involve functional abnormalities in neuronal activity, microvascular function, glia-neuron interaction, and neuron-neuron communication in different functional areas (Tecchio et al., 2006; Onifer et al., 2007; Nardone et al., 2013; Carron et al., 2016; Urbin et al., 2017). Any of these abnormalities could potentially affect each other, and could all contribute to the alterations in rsfMRI signals. Even though the underlying neural mechanisms and driving force of the synchronized rsfMRI signal fluctuation are not fully understood, shared common inputs (e.g., from sub- 

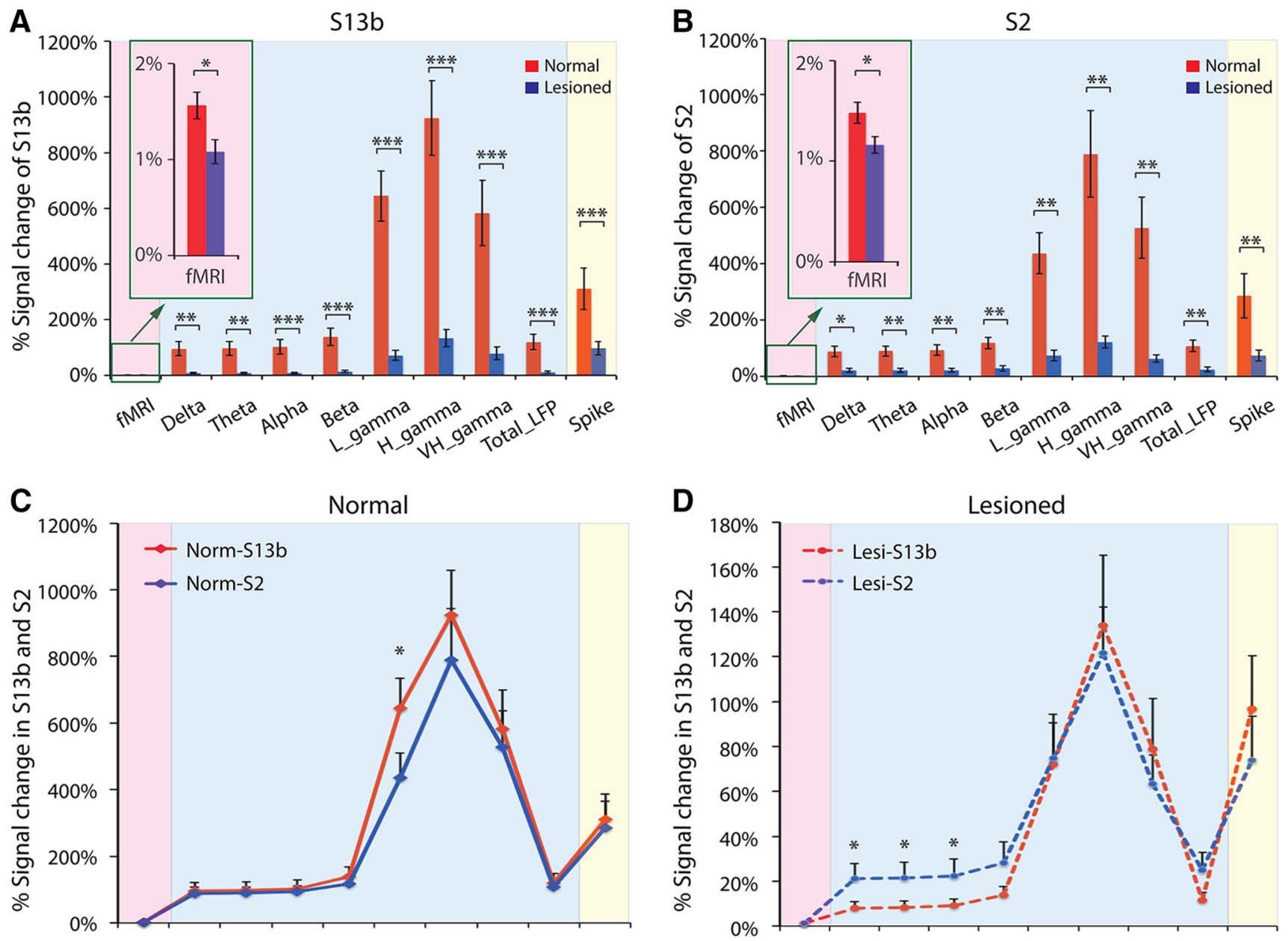

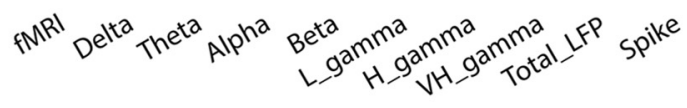

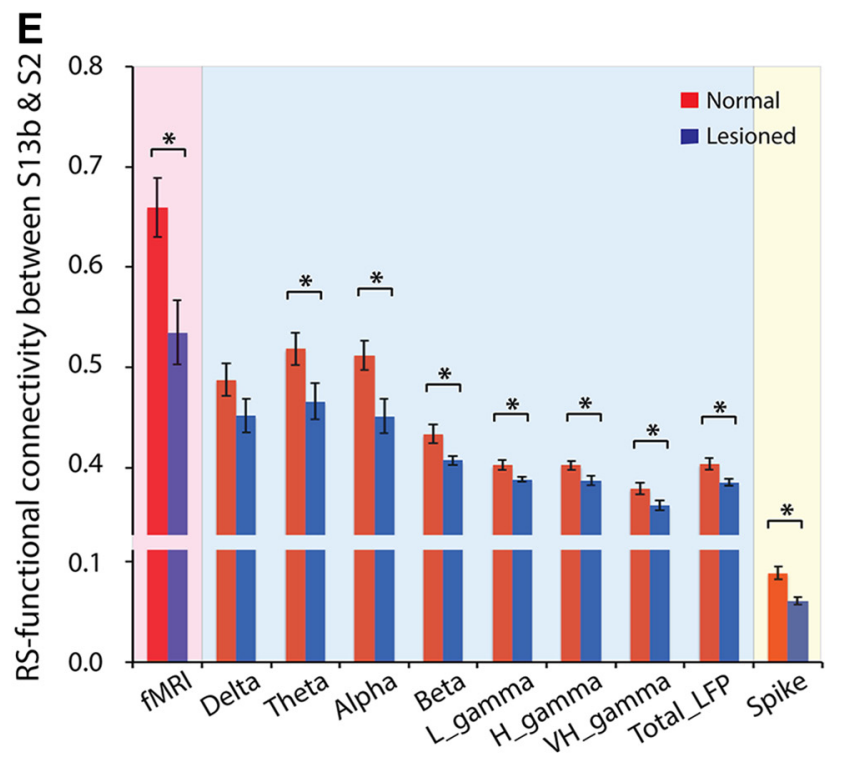

\section{$\mathbf{F}_{\text {Evoked signal \% and RS-FC effected by deafferentation }}$}

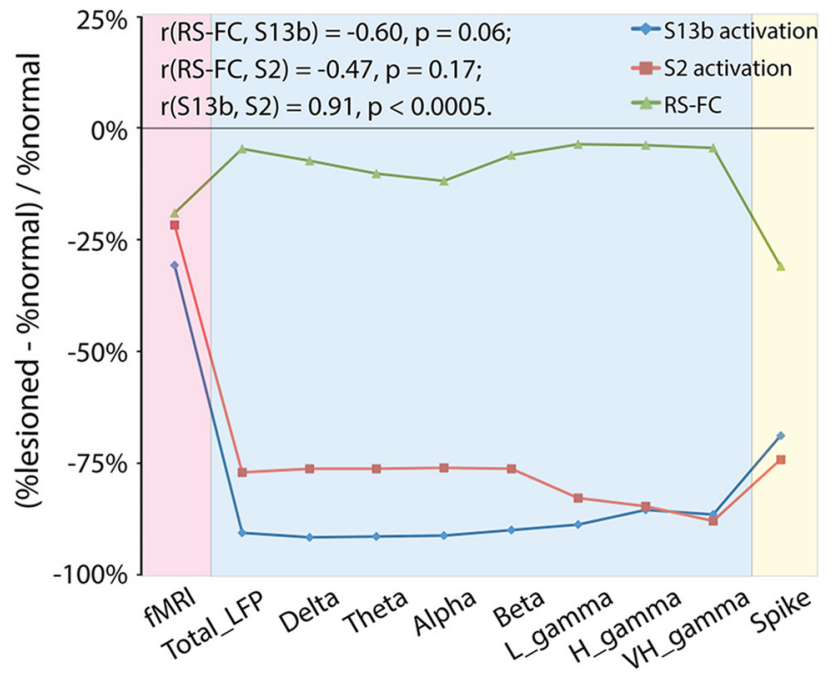

Figure 7. Group comparisons of $\mathrm{FC}$ between areas $3 \mathrm{~b}$ and $\mathrm{S} 2$, and those signal changes in normal and input-deprived monkeys used by fMRI, LFP, and spike activity. $A, B$, Signal changes of fMRI, LFP, and spike activity induced by tactile stimulus in areas $3 b(\boldsymbol{A})$ and $\mathrm{S2}(\boldsymbol{B})$ in normal versus input-deprived animals. Red columns represent normal condition. Blue columns represent lesioned condition. $\boldsymbol{C}, \boldsymbol{D}$, Signal changes of fMRI, LFP, and spike activity induced by tactile stimulus in area 3 b versus $S 2$ in normal $(\boldsymbol{C})$ and lesioned animals $(\boldsymbol{D})$. The $y$-axis scale bars in $\boldsymbol{C}$ and $\boldsymbol{D}$ are different. Red lines indicate area $3 \mathrm{~b}$. Blue lines indicate S2. $\boldsymbol{E}$, Summary of rsFC between areas $3 \mathrm{~b}$ and $\mathrm{S} 2$ in normal (red columns) versus lesioned (blue columns) animals derived from different signals. $\boldsymbol{F}$, Relationship between evoked signal change in areas $3 \mathrm{~b}$ and $\mathrm{S} 2$, and $\mathrm{FC}$ change between areas $3 \mathrm{~b}$ and $\mathrm{S} 2$, induced by deafferentation. ${ }^{*} p<0.05$ (unpaired $t$ test between normal and lesioned group). ${ }^{* *} p<0.005$ (unpaired $t$ test between normal and lesioned group). ${ }^{* *} p<0.0005$ (unpaired $t$ test between normal and lesioned group). 

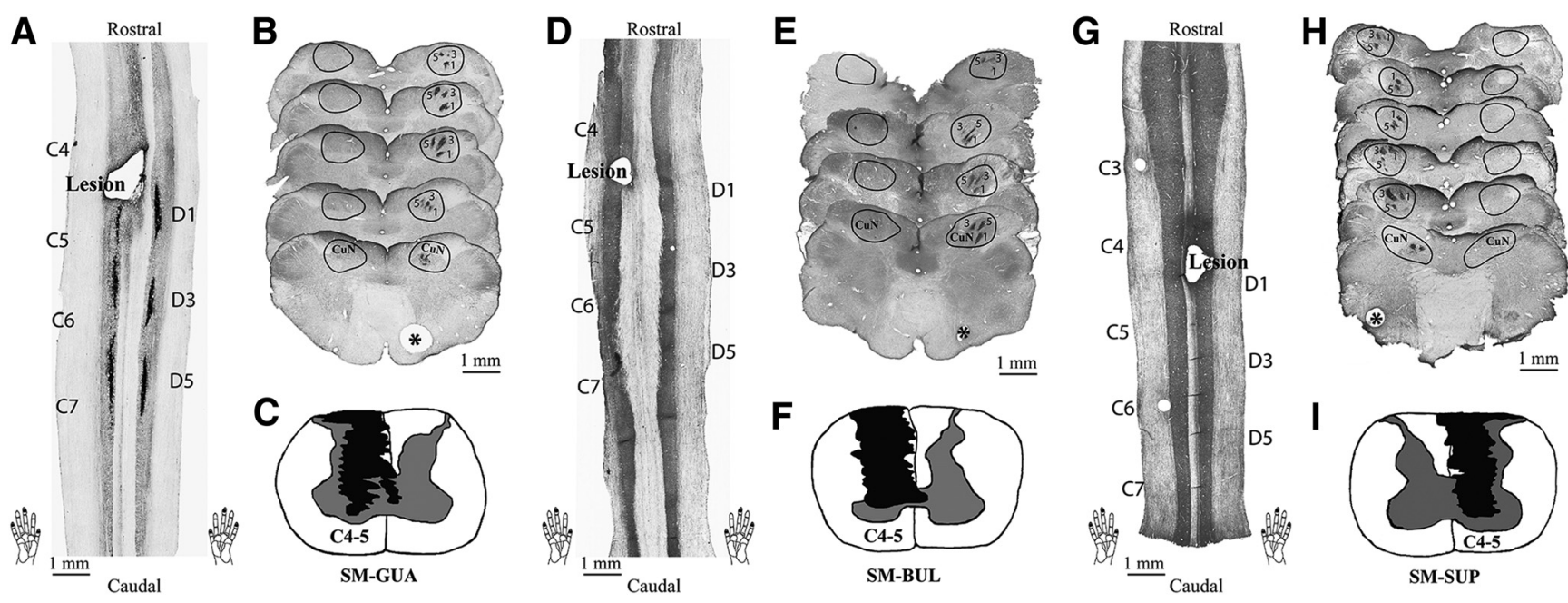

Figure 8. Histological evaluation of the level and extent of the lesion in the spinal cord, and CTB-labeled terminations in the cuneate nucleus (CuN) of the brainstem in 3 monkeys ( $A-C$, SM-GUA; $D-F$, SM-BUL; and G-I, SM-SUP). A, D, G, One coronal section of the spinal cord showing the location and level of the lesion. Cervical segments $4-7$ (C4-C7) and the CTB injections sites on digits 1,3 , and 5 (bottom two hand inserts) are marked. $\mathbf{G}$, Two pin holes at $(3$ and D6 levels are visible. CTB stains are missing in coronal sections of cord (D, G). $\boldsymbol{B}, \boldsymbol{E}, \boldsymbol{H}$, Examples of axial CTBimmunoreacted sections through cuneate nuclei of the brainstem. The CuN on each section is outlined. Numbers 1,3 , and 5 indicate the CTB label clusters resulting from injections on digits 1,3 , and 5 , respectively. There are a few detectable (TB-labeled terminations on the lesioned side. *Normal sides. $C, F, I$, Reconstructed transverse sections of the spinal cord showing the extent of the lesion in black patches. The lesions were reconstructed from a series of horizontally cut sections.

cortical regions) or extensive corticocortical connections are two viable hypotheses (Schölvinck et al., 2010; Wang et al., 2013). Given the complex pathology of many disorders, it is desirable to selectively alter one key component while keeping all others constant. DCL at the high cervical spinal cord level exemplifies such an ideal model for understanding the role of thalamic inputs to the synchrony of fMRI and electrophysiological activities between two highly functionally related cortical areas (e.g., areas 3b and S2 here). The cortical input deprivation introduced by selective disruption of the ascending dorsal column afferent represents a relatively "controlled" pathological condition compared with other models, such as direct injury to the brain. In this DCL model, the degree of digit afferent disruption can be validated and quantified by subsequent histological tracer-tracking methods (Kaas and Collins, 2003; Qi et al., 2011; Wu et al., 2016). With these data from each animal, we are confident about the input deprivation status of digit representation regions from which data are collected. To overcome the variability in cortical stimulusresponse pattern changes (i.e., plastic reorganization) (for a detailed description, see Yang et al., 2015) after DCL, we used fMRI activation maps to guide our electrophysiology recordings. This experimental design permits us to directly examine the effects of input deprivation on the cortex during information processing in response to external stimuli and at resting states, and to determine how changes in these two different states relate to each other. Identification of somatotopically reorganized digit representation maps and observations of significantly reduced stimulus-evoked fMRI signal amplitude changes in input-deprived areas 3b and S2 weeks after DCL (Fig. 4) are consistent with previous studies (Min et al., 2015). One step further, we found significant, correlated reductions in LFP and spiking responses in corresponding fMRI responsive regions when identical stimulation was applied. Together, these observations confirm the deafferentation status of the cortical areas 3b and S2 in each animal, information that is essential for our subsequent examination of the $\mathrm{FC}$ alterations by three different types of resting-state signals (i.e., fMRI, LFP, and spiking).
The relationship between stimulus-evoked response reduction and rsFC weakening in input-deprived cortex

The correlated stimulus-evoked response reduction indicates that area $3 \mathrm{~b}$ and $\mathrm{S} 2$ neurons lost their primary ascending inputs, and the rsFC weakening represents compromised interareal communications through loss of synchrony in spontaneous neuronal activities. Our findings support that, at least at this mesoscale, the common inputs of the area 3b-S2 circuit are an important driving force of the synchronized resting-state signal fluctuation. Reductions in rsfMRI signal correlation covary with decreased rsFC calculated with LFP and spiking signals. This finding supports the validity of using rsfMRI and rsFC to probe comprised interareal and interneuronal communication and functional connection.

Interestingly, the degrees of stimulus-response reductions and rsFC weakening captured by different signals varied. This observation indicates that the different signals may reflect different aspects of neuronal processes occurring after deafferentation. After nearly complete DCL (97\% disruption of ascending afferent from digits), stimulus-evoked LFP signals showed the largest signal drop $(\sim 80 \%)$, whereas the fMRI signal showed the smallest reduction $(\sim 25 \%)$. At resting state, spiking activity showed the biggest rsFC weakening $(\sim 30 \%)$, whereas the LFP exhibited the smallest reduction $(\sim 10 \%)$. The largest stimulus-state-dependent differences were observed in LFP signals (Fig. $7 F$ ). Based on the known signal origin differences of fMRI, LFP, and spike, the observed differences in the degrees of reduction in stimulus responsiveness versus rsFC captured by different signals support the presence of regained or alternative driving forces for the synchronized rsfMRI fluctuation in input-deprived and reorganized cortex. It is known that, during normal cortical processing of incoming peripheral inputs (e.g., tactile information), changes in fMRI signals correlate more closely with changes LFP signals, which predominantly reflect integration of synaptic activities (Okun et al., 2010; Ojemann et al., 2013; Ness et al., 2016). Spiking activity, on the other hand, reflects the output signals for interareal communication. Although all three signals reflect the compromised interareal functional connection, the changes in 
their respective FC measures may be weighted toward different neuronal processes (Berens et al., 2008; Boynton, 2011; Baranauskas et al., 2012; Barbieri et al., 2014). The largest drop in LFP response magnitude can be explained by the $97 \%$ deprivation of thalamic input signals. The biggest FC weakening captured by spiking synchrony suggests that spiking synchrony is a more sensitive indicator for the loss of efficacy in FC between neurons of inputdeprived cortical areas. It is also likely that altered and weakened input signal from alternative ascending pathways, partial restoration of dorsal column inputs through sprouting of connections of spared fibers at the spinal cord, synaptic efficacy alteration, and interhemisphere compensation may all be contributing to adequately maintain fairly strong FC between regions (for review, see Rossini and Pauri, 2000). Whether the interareal FC underwent a dynamic alteration, and how to relate this to behavioral recovery, remains to be determined. It will be important for future studies to tease out the contributions of the incoming synaptic integration versus outgoing spiking activity to the different rsFC measures, and ultimately to their relationships with the functional and behavioral recovery. One final note is that all the animals studied exhibited full recovery of hand use at the time microelectrode electrophysiology data were collected. A longitudinal recording study at different time points after DCL would provide the time-dependent information for understanding the contributions of different plastic processes to FC measurement alternation and their relationships to behavioral recovery (Yang et al., 2014).

\section{What have we learned from the very similar reductions in area $3 b$ versus $S 2$ in stimulation and resting conditions?} Another interesting finding of the present study is the very similar reductions in cortical responsiveness and $\mathrm{rsFC}$ in areas $3 \mathrm{~b}$ and S2 after DCL. Based on anatomical connection, neuronal response, and receptive field properties, S2 is considered to be a high-order area for fine touch processing, a predominant function for dorsal column afferents. S2 cortex receives direct cortical inputs from S1, especially from area 3b (Kaas et al., 1984; Cusick et al., 1985; Pons and Kaas, 1985, 1986). The strong rsFC between areas 3b and S2 in the normal monkey brain, as evidenced by the high synchrony of their low-frequency BOLD and LFP signals and correlated spiking activities, further supports their engagement in hand touch function and the notion that anatomically connected regions exhibit high FC at rest. If the corticocortical connections between areas $3 \mathrm{~b}$ and $\mathrm{S} 2$ contributed to the rsFC to the same extent as thalamic inputs, we would expect different reductions in rsFC across different signals after DCL. Indeed, the very similar rsFC reductions in areas $3 \mathrm{~b}$ and $\mathrm{S} 2$ in this experimental model suggest that the thalamic inputs are the main driving force for the synchronized fMRI signal and neuronal electrical activity within mesoscale circuits at rest.

In conclusion, our data demonstrate that cortical areas exhibiting compromised processing of peripheral sensory inputs also show weakened rsFC. Reduction in rsFC revealed by rsfMRI signals is functionally relevant and is reflective of disrupted FC measured by LFP and spiking signals. Our findings validate the use of rsfMRI to probe FC alterations in deafferented and inputdeprived cortices. Different signals, however, have different sensitivity in detecting alterations in interareal FC. In the context of current debate on the functional and clinical relevance of FC alterations (enhancement or reduction), our results support that a rsFC reduction between two mesoscale cortical regions relates to behavioral deficit.

\section{References}

Baranauskas G, Maggiolini E, Vato A, Angotzi G, Bonfanti A, Zambra G, Spinelli A, Fadiga L (2012) Origins of $1 / \mathrm{f} 2$ scaling in the power spectrum of intracortical local field potential. J Neurophysiol 107:984-994. CrossRef Medline

Barbieri F, Mazzoni A, Logothetis NK, Panzeri S, Brunel N (2014) Stimulus dependence of local field potential spectra: experiment versus theory. J Neurosci 34:14589-14605. CrossRef Medline

Berens P, Keliris GA, Ecker AS, Logothetis NK, Tolias AS (2008) Feature selectivity of the gamma-band of the local field potential in primate primary visual cortex. Front Neurosci 2:199-207. CrossRef Medline

Biswal B, Yetkin FZ, Haughton VM, Hyde JS (1995) Functional connectivity in the motor cortex of resting human brain using echo-planar MRI. Magn Reson Med 34:537-541. CrossRef Medline

Boynton GM (2011) Spikes, BOLD, attention, and awareness: a comparison of electrophysiological and fMRI signals in V1. J Vis 11:12. CrossRef Medline

Carron SF, Alwis DS, Rajan R (2016) Traumatic brain injury and neuronal functionality changes in sensory cortex. Front Syst Neurosci 10:47. CrossRef Medline

Chen LM, Friedman RM, Ramsden BM, LaMotte RH, Roe AW (2001) Finescale organization of SI (area $3 \mathrm{~b}$ ) in the squirrel monkey revealed with intrinsic optical imaging. J Neurophysiol 86:3011-3029. Medline

Chen LM, Qi HX, Kaas JH (2012) Dynamic reorganization of digit representations in somatosensory cortex of nonhuman primates after spinal cord injury. J Neurosci 32:14649-14663. CrossRef Medline

Chen LM, Mishra A, Yang PF, Wang F, Gore JC (2015) Injury alters intrinsic functional connectivity within the primate spinal cord. Proc Natl Acad Sci U S A 112:5991-5996. CrossRef Medline

Choe AS, Belegu V, Yoshida S, Joel S, Sadowsky CL, Smith SA, van Zijl PC, Pekar JJ, McDonald JW (2013) Extensive neurological recovery from a complete spinal cord injury: a case report and hypothesis on the role of cortical plasticity. Front Hum Neurosci 7:290. CrossRef Medline

Cusick CG, Steindler DA, Kaas JH (1985) Corticocortical and collateral thalamocortical connections of postcentral somatosensory cortical areas in squirrel monkeys: a double-labeling study with radiolabeled wheatgerm agglutinin and wheatgerm agglutinin conjugated to horseradish peroxidase. Somatosens Res 3:1-31. CrossRef Medline

Eckhorn R, Bauer R, Jordan W, Brosch M, Kruse W, Munk M, Reitboeck H) (1988) Coherent oscillations: a mechanism of feature linking in the visual cortex? Multiple electrode and correlation analyses in the cat. Biol Cybern 60:121-130. CrossRef Medline

Ekstrom A (2010) How and when the fMRI BOLD signal relates to underlying neural activity: the danger in dissociation. Brain Res Rev 62:233244. CrossRef Medline

Fox MD, Greicius M (2010) Clinical applications of resting state functional connectivity. Front Syst Neurosci 4:19. CrossRef Medline

Jain N, Qi HX, Collins CE, Kaas JH (2008) Large-scale reorganization in the somatosensory cortex and thalamus after sensory loss in macaque monkeys. J Neurosci 28:11042-11060. CrossRef Medline

Kaas JH (1993) The functional organization of somatosensory cortex in primates. Ann Anat 175:509-518. CrossRef Medline

Kaas JH, Collins CE (2003) Anatomic and functional reorganization of somatosensory cortex in mature primates after peripheral nerve and spinal cord injury. Adv Neurol 93:87-95. Medline

Kaas JH, Nelson RJ, Sur M, Dykes RW, Merzenich MM (1984) The somatotopic organization of the ventroposterior thalamus of the squirrel monkey, Saimiri sciureus. J Comp Neurol 226:111-140. CrossRef Medline

Krubitzer LA, Kaas JH (1992) The somatosensory thalamus of monkeys: cortical connections and a redefinition of nuclei in marmosets. J Comp Neurol 319:123-140. CrossRef Medline

Logothetis NK, Pauls J, Augath M, Trinath T, Oeltermann A (2001) Neurophysiological investigation of the basis of the fMRI signal. Nature 412: 150-157. CrossRef Medline

Min YS, Park JW, Jin SU, Jang KE, Nam HU, Lee YS, Jung TD, Chang Y (2015) Alteration of resting-state brain sensorimotor connectivity following spinal cord injury: a resting-state functional magnetic resonance imaging study. J Neurotrauma 32:1422-1427. CrossRef Medline

Mukamel R, Gelbard H, Arieli A, Hasson U, Fried I, Malach R (2005) Coupling between neuronal firing, field potentials, and fMRI in human auditory cortex. Science 309:951-954. CrossRef Medline

Nardone R, Höller Y, Brigo F, Seidl M, Christova M, Bergmann J, Golaszewski 
S, Trinka E (2013) Functional brain reorganization after spinal cord injury: systematic review of animal and human studies. Brain Res 1504: 58-73. CrossRef Medline

Ness TV, Remme MW, Einevoll GT (2016) Active subthreshold dendritic conductances shape the local field potential. J Physiol 594:3809-3825. CrossRef Medline

Ojemann GA, Ojemann J, Ramsey NF (2013) Relation between functional magnetic resonance imaging (fMRI) and single neuron, local field potential (LFP) and electrocorticography (ECoG) activity in human cortex. Front Hum Neurosci 7:34. CrossRef Medline

Okun M, Naim A, Lampl I (2010) The subthreshold relation between cortical local field potential and neuronal firing unveiled by intracellular recordings in awake rats. J Neurosci 30:4440-4448. CrossRef Medline

Onifer SM, Nunn CD, Decker JA, Payne BN, Wagoner MR, Puckett AH, Massey JM, Armstrong J, Kaddumi EG, Fentress KG, Wells MJ, West RM, Calloway CC, Schnell JT, Whitaker CM, Burke DA, Hubscher CH (2007) Loss and spontaneous recovery of forelimb evoked potentials in both the adult rat cuneate nucleus and somatosensory cortex following contusive cervical spinal cord injury. Exp Neurol 207:238-247. CrossRef Medline

Plewnia C, Rilk AJ, Soekadar SR, Arfeller C, Huber HS, Sauseng P, Hummel F, Gerloff C (2008) Enhancement of long-range EEG coherence by synchronous bifocal transcranial magnetic stimulation. Eur J Neurosci 27: 1577-1583. CrossRef Medline

Pons TP, Kaas JH (1985) Connections of area 2 of somatosensory cortex with the anterior pulvinar and subdivisions of the ventroposterior complex in macaque monkeys. J Comp Neurol 240:16-36. CrossRef Medline

Pons TP, Kaas JH (1986) Corticocortical connections of area 2 of somatosensory cortex in macaque monkeys: a correlative anatomical and electrophysiological study. J Comp Neurol 248:313-335. CrossRef Medline

Qi HX, Chen LM, Kaas JH (2011) Reorganization of somatosensory cortical areas $3 \mathrm{~b}$ and 1 after unilateral section of dorsal columns of the spinal cord in squirrel monkeys. J Neurosci 31:13662-13675. CrossRef Medline

Raichle ME (2010) Two views of brain function. Trends Cogn Sci 14:180190. CrossRef Medline

Rao JS, Ma M, Zhao C, Zhang AF, Yang ZY, Liu Z, Li XG (2014) Fractional amplitude of low-frequency fluctuation changes in monkeys with spinal cord injury: a resting-state fMRI study. Magn Reson Imaging 32:482-486. CrossRef Medline

Reed JL, Pouget P, Qi HX, Zhou Z, Bernard MR, Burish MJ, Haitas J, Bonds $\mathrm{AB}$, Kaas JH (2008) Widespread spatial integration in primary somatosensory cortex. Proc Natl Acad Sci U S A 105:10233-10237. CrossRef Medline

Reed JL, Pouget P, Qi HX, Zhou Z, Bernard MR, Burish MJ, Kaas JH (2012) Effects of spatiotemporal stimulus properties on spike timing correlations in owl monkey primary somatosensory cortex. J Neurophysiol 108:33533369. CrossRef Medline

Rossini PM, Pauri F (2000) Neuromagnetic integrated methods tracking human brain mechanisms of sensorimotor areas 'plastic' reorganisation. Brain Res Brain Res Rev 33:131-154. CrossRef Medline
Rotarska-Jagiela A, van de Ven V, Oertel-Knöchel V, Uhlhaas PJ, Vogeley K, Linden DE (2010) Resting-state functional network correlates of psychotic symptoms in schizophrenia. Schizophr Res 117:21-30. CrossRef Medline

Schölvinck ML, Maier A, Ye FQ, Duyn JH, Leopold DA (2010) Neural basis of global resting-state fMRI activity. Proc Natl Acad Sci U S A 107:1023810243. CrossRef Medline

Shi Z, Wu R, Yang PF, Wang F, Wu TL, Mishra A, Chen LM, Gore JC (2017) High spatial correspondence at a columnar level between activation and resting state fMRI signals and local field potentials. Proc Natl Acad Sci U S A 114:5253-5258. CrossRef Medline

Tecchio F, Zappasodi F, Pasqualetti P, Tombini M, Caulo M, Ercolani M, Rossini PM (2006) Long-term effects of stroke on neuronal rest activity in rolandic cortical areas. J Neurosci Res 83:1077-1087. CrossRef Medline

Urbin MA, Ozdemir RA, Tazoe T, Perez MA (2017) Spike-timing-dependent plasticity in lower-limb motoneurons after human spinal cord injury. J Neurophysiol 118:2171-2180. CrossRef Medline

van den Heuvel MP, Hulshoff Pol HE (2010) Exploring the brain network: a review on resting-state fMRI functional connectivity. Eur Neuropsychopharmacol 20:519-534. CrossRef Medline

Wang Z, Qi HX, Kaas JH, Roe AW, Chen LM (2013) Functional signature of recovering cortex: dissociation of local field potentials and spiking activity in somatosensory cortices of spinal cord injured monkeys. Exp Neurol 249:132-143. CrossRef Medline

Wang Z, Yang Q, Chen LM (2017) Abnormal dynamics of cortical resting state functional connectivity in chronic headache patients. Magn Reson Imaging 36:56-67. CrossRef Medline

Wilson GH 3rd, Yang PF, Gore JC, Chen LM (2016) Correlated interregional variations in low frequency local field potentials and resting state BOLD signals within S1 cortex of monkeys. Hum Brain Mapp 37:27552766. CrossRef Medline

Wu R, Su L, Yang PF, Chen LM (2016) Altered spatiotemporal dynamics of cortical activation to tactile stimuli in somatosensory area $3 \mathrm{~b}$ and area 1 of monkeys after spinal cord injury. eNeuro 3:ENEURO.0095-16.2016. CrossRef Medline

Wu R, Wang F, Yang PF, Chen LM (2017) High-resolution functional MRI identified distinct global intrinsic functional networks of nociceptive posterior insula and S2 regions in squirrel monkey brain. Neuroimage 155: 147-158. CrossRef Medline

Yang PF, Qi HX, Kaas JH, Chen LM (2014) Parallel functional reorganizations of somatosensory areas $3 \mathrm{~b}$ and 1 , and $\mathrm{S} 2$ following spinal cord injury in squirrel monkeys. J Neurosci 34:9351-9363. CrossRef Medline

Yang PF, Wang F, Chen LM (2015) Differential fMRI activation patterns to noxious heat and tactile stimuli in the primate spinal cord. J Neurosci 35:10493-10502. CrossRef Medline

Yang Q, Wang Z, Yang L, Xu Y, Chen LM (2017) Cortical thickness and functional connectivity abnormality in chronic headache and low back pain patients. Hum Brain Mapp 38:1815-1832. CrossRef Medline 